\title{
TU/e EmonOWEN

\section{A Multi-Cue Bioreactor to Evaluate the Inflammatory and Regenerative Capacity of Biomaterials under Flow and Stretch}

Citation for published version (APA):

Koch, S. E., van Haaften, E. E., Wissing, T. B., Cuypers, L. A. B., Bulsink, J., Bouten, C. V. C., Kurniawan, N. A., \& Smits, A. I. P. M. (2020). A Multi-Cue Bioreactor to Evaluate the Inflammatory and Regenerative Capacity of Biomaterials under Flow and Stretch. Journal of Visualized Experiments (JoVE), 166, [e61824]. https://doi.org/10.3791/61824

\section{Document license:}

TAVERNE

DOI:

$10.3791 / 61824$

Document status and date:

Published: 10/12/2020

\section{Document Version:}

Publisher's PDF, also known as Version of Record (includes final page, issue and volume numbers)

\section{Please check the document version of this publication:}

- A submitted manuscript is the version of the article upon submission and before peer-review. There can be important differences between the submitted version and the official published version of record. People interested in the research are advised to contact the author for the final version of the publication, or visit the $\mathrm{DOI}$ to the publisher's website.

- The final author version and the galley proof are versions of the publication after peer review.

- The final published version features the final layout of the paper including the volume, issue and page numbers.

Link to publication

\section{General rights}

Copyright and moral rights for the publications made accessible in the public portal are retained by the authors and/or other copyright owners and it is a condition of accessing publications that users recognise and abide by the legal requirements associated with these rights.

- Users may download and print one copy of any publication from the public portal for the purpose of private study or research.

- You may not further distribute the material or use it for any profit-making activity or commercial gain

- You may freely distribute the URL identifying the publication in the public portal.

If the publication is distributed under the terms of Article 25fa of the Dutch Copyright Act, indicated by the "Taverne" license above, please follow below link for the End User Agreement:

www.tue.nl/taverne

Take down policy

If you believe that this document breaches copyright please contact us at:

openaccess@tue.nl

providing details and we will investigate your claim. 


\section{A Multi-Cue Bioreactor to Evaluate the Inflammatory and Regenerative Capacity of Biomaterials under Flow and Stretch}

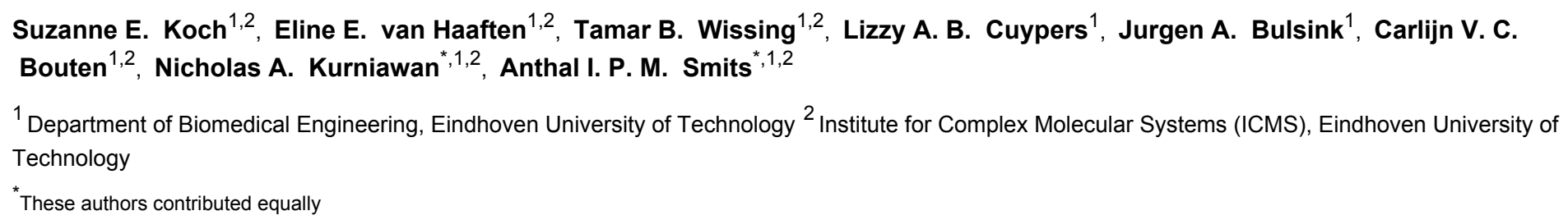

\section{Corresponding Authors}

Nicholas A. Kurniawan

n.a.kurniawan@tue.nl

Anthal I. P. M. Smits

a.i.p.m.smits@tue.nl

\section{Citation}

Koch, S.E., van Haaften, E.E., Wissing, T.B., Cuypers, L.A.B., Bulsink, J.A., Bouten, C.V.C., Kurniawan, N.A., Smits, A.I.P.M. A Multi-Cue Bioreactor to Evaluate the Inflammatory and Regenerative Capacity of Biomaterials under Flow and Stretch. J. Vis. Exp. (166), e61824, doi:10.3791/61824 (2020).

\section{Date Published}

December 10, 2020

DOI

$10.3791 / 61824$

\section{URL}

jove.com/video/61824

\section{Abstract}

The use of resorbable biomaterials to induce regeneration directly in the body is an attractive strategy from a translational perspective. Such materials induce an inflammatory response upon implantation, which is the driver of subsequent resorption of the material and the regeneration of new tissue. This strategy, also known as in situ tissue engineering, is pursued to obtain cardiovascular replacements such as tissue-engineered vascular grafts. Both the inflammatory and the regenerative processes are determined by the local biomechanical cues on the scaffold (i.e., stretch and shear stress). Here, we describe in detail the use of a custom-developed bioreactor that uniquely enables the decoupling of stretch and shear stress on a tubular scaffold. This allows for the systematic and standardized evaluation of the inflammatory and regenerative capacity of tubular scaffolds under the influence of wellcontrolled mechanical loads, which we demonstrate on the basis of a dynamic coculture experiment using human macrophages and myofibroblasts. The key practical steps in this approach-the construction and setting up of the bioreactor, preparation of the scaffolds and cell seeding, application and maintenance of stretch and shear flow, and sample harvesting for analysis—are discussed in detail.

\section{Introduction}

Cardiovascular tissue engineering (TE) is being pursued as an alternative treatment option to the currently used permanent cardiovascular prostheses (e.g., vascular grafts, heart valve replacements), which are suboptimal for large cohorts of patients ${ }^{1,2,3,4}$. Much sought-after applications include tissue-engineered vascular grafts (TEVGs) $)^{5,6}$ and 
heart valves $(\mathrm{TEHVs})^{7,8}$. Most often, cardiovascular TE methodologies make use of resorbable biomaterials (either natural or synthetic) that serve as an instructive scaffold for the new tissue to be formed. The formation of new tissue can either be engineered completely in vitro, by seeding the scaffold with cells and culturing in a bioreactor prior to implantation (in vitro $T E)^{9,10,11}$, or directly in situ, in which the synthetic scaffold is implanted without pre-culturing in order to induce the formation of new tissue directly in the body (in situ TE) $)^{12,13,14}$. For both in vitro and in situ cardiovascular TE approaches, successful functional regeneration is dominantly dependent on both the host immune response to the implanted construct and appropriate biomechanical loading.

The importance of biomechanical loading for cardiovascular TE is well-acknowledged ${ }^{15}$. In the case of cardiovascular implants, the cells that populate the scaffold are exposed to cyclic stretch and shear stresses that arise as a result of the hemodynamic environment. Numerous studies have reported the stimulatory effect of (cyclic) stretch on the formation of matrix components, such as collagen $16,17,18,19$, glycosaminoglycans $(G A G s)^{20}$, and elastin $^{21,22}$, by various cell types. For example, Huang et al. demonstrated that biaxial stretch elevated the deposition and organization of collagen and elastin in in vitro TEVGs by using a vascular bioreactor ${ }^{23}$. While the emphasis typically lies on stretch as the dominant load, these studies often make use of flow-driven bioreactors in which the sample is also exposed to shear flow. Although relatively little is known about the isolated influence of shear stresses on tissue formation and inflammation in 3D, some data are available. For example, Hinderer et al. and Eoh et al. demonstrated that shear flow, in addition to a 3D scaffold microstructure, was important for the formation of mature elastin by human vascular smooth muscle cells in an in vitro model system ${ }^{24,25}$. Altogether, these findings illustrate the relevance of both cyclic stretch and shear stress for cardiovascular TE.

Another important determinant for the success or failure of TE implants is the host's immune response to the implanted graft ${ }^{26}$. This is particularly important for materialdriven in situ TE strategies, which actually rely on the acute inflammatory response to the scaffold to kickstart the subsequent processes of cellular influx and endogenous tissue formation and remodeling ${ }^{27}$. The macrophage is a critical initiator of functional tissue regeneration, which has been shown by multiple studies ${ }^{28,29,30}$. Analogous to wound healing, the regeneration of tissue is governed by paracrine signaling between macrophages and tissue-producing cells such as fibroblasts and myofibroblasts $31,32,33$. In addition to coordinating new tissue deposition, macrophages are involved in the active resorption of foreign scaffold material $^{34,35}$. As such, the in vitro macrophage response to a biomaterial has been identified as a predictive parameter for the in vivo success of implants $36,37,38$.

The macrophage response to an implanted scaffold is dependent on scaffold design features such as material composition and microstructure $35,39,40$. In addition to scaffold properties, the macrophage response to a scaffold and their crosstalk with myofibroblasts is also impacted by hemodynamic loads. For example, cyclic stretch was shown to be an important modulator of macrophage phenotype $41,42,43,44$ and the secretion of cytokines $43,44,45,46$ in 3D electrospun scaffolds. Using a coculture system of macrophages and vascular smooth muscle cells, Battiston et al. demonstrated that the presence of macrophages led to increased levels of elastin and GAGs and that moderate levels of cyclic stretch (1.07-1.10) stimulated 
the deposition of collagen I and elastin ${ }^{47}$. In previous works, we have demonstrated that shear stress is an important determinant for monocyte recruitment into 3D electrospun scaffolds 48,49 , and that both shear stress and cyclic stretch impact the paracrine signaling between human monocytes and mesenchymal stromal cells ${ }^{50}$. Fahy et al. demonstrated that shear flow increased the secretion of pro-inflammatory cytokines by human monocytes ${ }^{51}$.

Taken together, the above evidence shows that an adequate understanding of and control over hemodynamic loads is crucial for cardiovascular TE, and that it is important to consider the inflammatory response to achieve this. Numerous bioreactors have been described previously for the in vitro $52,53,54,55,56,57,58$ or ex vivo $59,60,61$ culture of cardiovascular tissues. However, all these systems are designed to mimic the physiological hemodynamic loading conditions as much as possible. While this is highly valuable for the purpose of creating cardiovascular tissues in vitro or maintaining ex vivo cultures, such systems do not allow for systematic studies into the individual effects of individual cues. This is because the application of both cyclic stretch and shear stress in these bioreactors is driven by the same pressurized flow, which intrinsically links them. While microsystems that allow for accurate multi-cue mechanical manipulation have been described for $2 D$ substrates $^{62}$ or 3D hydrogel setups 63,64 , such setups do not allow for the incorporation of elastomeric 3D biomaterial scaffolds.

Here, we present the application of a tubular bioreactor system that uniquely enables the decoupling of shear stress and cyclic stretch and helps to mechanistically investigate their individual and combined effects. This system allows for testing of a broad variety of tissue engineered vascular grafts (e.g., synthetic or natural origin, different micro-architecture, various porosities). To effectively decouple the application of shear stress and stretch, the key concepts that the bioreactor uses are (1) separation of the control of shear stress and stretch using distinct pump systems and (2) stimulation of the scaffolds in an 'inside-out' manner with computationally driven dimensions. Flow is applied on the outside surface of the tubular scaffold through the use of a flow pump, whereas circumferential stretch of the scaffold is induced by expanding a silicone tube on which the scaffold is mounted through the use of a separate strain pump. The dimensions of the silicone tube and the glass tube that contains the construct are carefully chosen and validated using computational fluid dynamics simulations, to ensure that the shear stress on the scaffold (due to flow) and the circumferential stretch (due to tube expansion) do not significantly affect each other. This inside-out design has several practical rationales. If stretch is applied by the luminal fluid pressure (similar to physiological loading), it inherently requires the sample design to be leakfree. In addition, the pressure required to stretch the sample would be completely determined by the sample stiffness, which may vary between samples and within a sample over time, making it difficult to control the stretch. This bioreactor mounts the tissue engineered graft around a silicone tube and allows for wall shear stress (WSS) application on the outer wall of the graft and pressurizes the graft from the inside. This way, equal loading conditions between samples and within samples over time can be ensured, and moreover, the samples are allowed to be leaky, as is common for porous vascular scaffolds ${ }^{19}$. This inside-out bioreactor is specifically intended for systematic studies on the effects of shear and/ or stretch, rather than the engineering of a native-like blood vessel in vitro, for which traditional vascular bioreactor setups are more suitable. See Figure 1A-B for the bioreactor design drawings, and its corresponding Table 1 for a functional 
description and rationale behind the main components of the bioreactor.

The use of the bioreactor is demonstrated on the basis of a series of recent studies by our group in which we investigated the individual and combined influences of shear stress and cyclic stretch on inflammation and tissue formation in resorbable electrospun scaffolds for in situ cardiovascular tissue $19,43,44$. To that end, we used human macrophages and myofibroblasts either in mono- or in co-culture to simulate the various phases of the in situ regenerative cascade. We have demonstrated that cytokine secretion by human macrophages is distinctly impacted by both cyclic stretch and shear stress, affecting the matrix deposition and organization by human myofibroblasts in these scaffolds, both via paracrine signaling and direct contact $^{19,43,44}$. Notably, these studies revealed that in the case of combined application of shear stress and stretch, the effects on tissue formation and inflammation are either dominated by one of the two loads, or there are synergistic effects of both loads. These findings illustrate the relevance of decoupling both loads to gain a better understanding of the contribution of the mechanical environment on TE processes. This understanding can be applied to systematically optimize scaffold design parameters in relevant hemodynamic loading regimes. In addition, the mechanistic data from such wellcontrolled environments may serve as input for numerical models that are being developed to predict the course of in situ tissue remodeling, as recently reported for TEVGs ${ }^{65}$ or TEHVs ${ }^{66}$, to further improve predictive capacity.

\section{Protocol}

In the studies described in this protocol, primary human macrophages isolated from peripheral blood buffy coats and human myofibroblasts isolated from the saphenous vein after coronary by-pass surgery have been used ${ }^{44}$. The buffy coats were obtained from healthy, anonymized volunteers who provided written informed consent, which was approved by the Sanquin Research Institutional Medical Ethical Committee. The use of human vena saphena cells (HVSCs) was in accordance to the "Code Proper Secondary Use of Human Tissue" developed by the Federation of Medical Societies (FMWV) in the Netherlands.

\section{General Preparations and Required Actions Before Setting Up the Bioreactor}

NOTE: For details on the respective isolation and culturing protocols, please refer to earlier work ${ }^{19,43,44}$. All calculations in the protocol are given as examples for a co-culture experiment with monocytes and myofibroblasts, seeded in 8 hemodynamically loaded scaffolds and 2 static controls $(n=10)$.

1. Start cell isolation and cell culture. The seeding densities for the co-cultured samples of monocytes and myofibroblasts (with a seeding ratio of $2: 1$ ) are $30 \times$ $10^{6}$ monocytes $/ \mathrm{cm}^{3}$ and $15 \times 10^{6}$ myofibroblasts $/ \mathrm{cm}^{3}$, respectively.

NOTE: The electrospun material has a high porosity $(>90 \%)$. To estimate the required number of cells per graft, the volume of the scaffold is calculated with the formula for volume of a hollow cylinder: $\pi^{*}$ (thickness) ${ }^{2 *}$ length $\approx 0.04 \mathrm{~cm}^{3}$. The total amount of cells per graft is $1.2 \times 10^{6}$ monocytes and $0.6 \times$ $10^{6}$ myofibroblasts. For 10 samples, at least $12 \times 10^{6}$ monocytes and $6 \times 10^{6}$ myofibroblasts are required; start with up to $\sim 10-15 \%$ more cells to account for possible pipetting errors. 
2. Degas the cell culture medium that will be used for experiments involving the bioreactor.

1. Prepare the medium for co-cultures, which consists of RPMI-1640:aDMEM (1:1), supplemented with $10 \%$ fetal bovine serum, $1 \%$ penicillin-streptomycin and $0.5 \%$ L-glutamine.

2. Place the medium overnight $(\mathrm{O} / \mathrm{N})$ in an incubator in a cell culture flask with filter cap to degas.

3. Replace the filter cap with an air-tight cap and store at $4{ }^{\circ} \mathrm{C}$.

4. Just before use, add $0.25 \mathrm{mg} / \mathrm{mL}$ L-ascorbic acid 2phosphate (vitamin C) to the medium.

NOTE: For calculations, the amount of medium required per flow culture chamber is $50 \mathrm{~mL}$. Refresh the medium three times per week; $25 \mathrm{~mL}$ old medium is replaced by $25 \mathrm{~mL}$ fresh medium. For 10 samples; after seeding, a total of $500 \mathrm{~mL}$ fresh medium is required, and for each subsequent medium change, a total of $250 \mathrm{~mL}$ fresh medium is used. Always prepare medium fresh, especially, vitamin C should be added just before changing the medium.

3. Prepare isotropic electrospun scaffolds $(3 \mathrm{~mm}$ luminal diameter, $200 \mu \mathrm{m}$ wall thickness) as described by Van Haaften et al. $^{19}$ (Figure 1G-I). In brief, tubular polycaprolactone bisurea (PCL-BU) scaffolds are produced by electrospinning from $15 \%(\mathrm{w} / \mathrm{w})$ chloroformpolymer solutions. The polymer solutions are electrospun at room temperature and $30 \%$ relative humidity, at a flow rate of $40 \mu \mathrm{L} / \mathrm{min}, 16 \mathrm{~cm}$ distance from the rotating cylindrical target ( $\varnothing 3 \mathrm{~mm}, 500 \mathrm{rpm}$ ), and an applied voltage of $16 \mathrm{kV}$ on the electrospinning nozzle and $-1 \mathrm{kV}$ on the target.
NOTE: Although PCL-BU grafts were used for these experiments, a broad variety of elastomeric tissue engineered grafts can be mounted in this bioreactor (e.g., of different synthetic or natural origin, different microarchitecture, different porosities)

1. Remove the electrospun scaffolds from the mandrel.

1. Make a small hole in the cap of a $15 \mathrm{~mL}$ tube to 'hold' the mandrel in the center and prevent it from touching the wall of the tube.

2. Place the mandrel with the electrospun scaffold in the falcon tube and fill it with deionized water.

3. Freeze the tubes $\mathrm{O} / \mathrm{N}$ at $-20^{\circ} \mathrm{C}$.

4. Place the tubes at room temperature (RT) and pull out the mandrels after a few minutes, leaving the electrospun grafts in the ice.

5. Let the ice thaw completely, remove the electrospun tube from the thawed water, and 'hang' to dry vertically for several hours. Make sure the scaffolds do not 'collapse' under their own weight.

2. Dry scaffolds under vacuum $\mathrm{O} / \mathrm{N}$.

3. Image a small sample of the electrospun grafts using scanning electron microscopy (SEM) to assess their microstructure (e.g., fiber morphology, fiber diameter). The grafts in the example studies have an isotropic fiber orientation and a fiber diameter of 5 $\mu \mathrm{m}$ (Figure 1H-I).

4. One day before starting the experiment, place the hydraulic reservoir filled with deionized water in the incubator. Close all eight connections for flow culture chambers with white Luer caps. Connect to the compressed air system and insert pressure sensor. Run 
the strain pump (see step 5.6) $\mathrm{O} / \mathrm{N}$ to allow for small expansion of the Teflon bellow.

NOTE: Make sure all necessary materials and equipment are cleaned and/or autoclaved (see Table of Materials, Comments/Description column for which materials are allowed to be autoclaved), according to the manufacturer's protocol or as described in steps 7.3-7.6.

5. Ensure sterile working conditions for the remainder of the protocol.

1. Perform steps $2-5.3$ (setting up the system), step 6.3 (medium change), and steps 7.1-7.2 (harvest of vascular constructs) in a sterile laminar flow cabinet.

2. Place materials that are not directly needed for the subsequent steps in closed Petri dishes to keep everything as clean as possible.

3. Clean or dry material surfaces regularly by soaking a paper tissue with $70 \%$ ethanol, and wipe the surfaces of the bioreactor components and the laminar flow cabinet.

\section{Setting Up the Bioreactor}

NOTE: Perform step 2 in a laminar flow cabinet.

1. Cut the electrospun scaffolds into tubes of approximately $25 \mathrm{~mm}$ in length, and document them before use (e.g., photograph for the length, weigh with balance for the initial mass).

2. Decontaminate the electrospun scaffolds.

1. Place the electrospun scaffolds tilted in a well plate or Petri dish, with one opening facing the ultraviolet (UV) light source, to enable UV light $(253.7 \mathrm{~nm})$ to illuminate the inside of the scaffolds.
2. Expose the electrospun scaffolds to UV light for 5 $\min$.

3. Turn all scaffolds and repeat the UV illumination for the other opening.

NOTE: After this step, only touch the electrospun scaffold when needed. Always use clean tweezers or clean gloves.

4. Take the glass tubes of the flow culture chambers that are stored in $70 \%$, wash the glass tubes in ultrapure water, dry, and place in a large, closed Petri dish.

NOTE: The following steps, especially steps $2.3-$ 2.5 , are ideally performed by two experimenters.

3. Mount the electrospun scaffolds onto the silicone tubing.

1. Attach the 5-0 prolene suture to one end of the silicone tubing by taking the suture through one side of the tube and out of the other, leaving two opposite taut sutures spanning the cross section of the tubing. Make a small knot on both sides of the tube while compressing the tube at the locus of the knots and leave approximately $10 \mathrm{~cm}$ of wire on both knots. Make a third knot at the end of the two $10 \mathrm{~cm}$ leftover wires.

1. Cut off the suture needle and all free threads that might stick out and damage the inside of the electrospun scaffold. Cut away the edges of the silicone tubing into a triangular shape to aid in pulling the silicone tubing through the electrospun scaffold.

2. Dip the electrospun scaffold in $30 \%$ ethanol (this serves as extra decontamination step and aids in sliding the electrospun scaffold over the silicon tubing) and place the electrospun scaffold over 
the free $10 \mathrm{~cm}$ wire. Experimenter $A$ stretches the silicone tubing by pulling gently on both the silicone tubing and the knot of the $10 \mathrm{~cm}$ suture wire, while experimenter $\mathrm{B}$ gently slides the electrospun scaffold over the silicone tubing using tweezers with a smooth inner tip to prevent damaging of the scaffolds.

3. Slowly release the stretch on the silicone tubing, while simultaneously smoothening the electrospun scaffold with tweezers. Dip the electrospun scaffold on the silicone tubing in ultrapure water two times.

NOTE: It is possible that some wrinkling of the electrospun scaffold occurs. This wrinkling will disappear during the applied pre-stretch right before fixing the scaffolds to the pressure conduits at step

\subsection{3.}

4. Repeat steps 2.3.2 and 2.3.3 for the other electrospun scaffolds. Depending on the length of the silicone tubing, multiple electrospun scaffolds can be mounted on the same silicone tubing.

5. When all electrospun scaffolds are mounted on the silicone tubing, cut the silicone tubing around the scaffolds, all to the same length $(5.5 \mathrm{~cm})$; at one side, close to the end of the electrospun scaffold, at the other side, leaving $\sim 2-3 \mathrm{~cm}$ of free silicone tubing.

4. Construct the bottom compartment of the flow culture chamber (Figure 1A-B).

1. Take the upper part of the bottom compartment containing the flow outlet, and close the flow outlet with a male Luer plug.

2. Push the pressure conduit with holes through the bottom compartment, and place a silicone O-ring around the lower end of the pressure conduit to prevent leakage. Screw the lower part of the bottom compartment to the upper part of the bottom compartment to secure the pressure conduit. Make sure that the lower engraved groove of the pressure conduit is approximately $3-5 \mathrm{~mm}$ above the edge of the adapter bushing of the bottom compartment; this will later 'hold' the tight knot of the suture wire, fixing the electrospun scaffold over the silicone tubing.

NOTE: If the pressure conduit can be easily maneuvered up and down, it indicates that the bottom compartment is not well secured. Repeat step 2.4.2 to prevent leakage in later stages (Figure 2D).

5. Secure the silicone tubing with the electrospun scaffold to the pressure conduit.

1. Pull the silicone tube with the electrospun scaffold over the pressure conduit.

2. Make a knot with the suture wire at the lower end of the electrospun scaffold at the location of the engraved groove on the pressure conduit. Make a second knot at the opposite side to tightly secure the silicone tubing with the electrospun graft.

CAUTION: This is a critical step. Make sure that the knot exactly 'falls' into the engraved groove of the pressure conduit to prevent leakage of the water from the hydraulic reservoir to the flow culture chambers. If not sure, try to tighten the suture wire at several positions, above or below the expected location of the groove, to ensure that the final knots are exactly at the engraved groove (Figure 2A).

3. Place the scissor clamp at the upper end of the silicone tube, and stretch the silicone tubing upwards (this will directly test the first knot, if it is possible 
to move the silicone tubing with the electrospun scaffold over the pressure conduit, it was not tightened well enough). With the pulling force, the silicone tubing is pre-stretched. To ensure that the silicone tubing is consistent among the different samples, attach a ruler to the scissor clamp. Pull the scissor clamp upwards until the lower end of the ruler reaches the height of the lower end of the scaffold.

NOTE: It is important to keep the pre-stretch in each sample roughly the same $(\sim 5 \%)$ for two reasons: (1) if silicone tubing is pre-stretched, it will result in more homogeneous expansion along the length of the sample when pressurized; (2) the pre-stretch will impact the mechanical properties of the silicone, therefore it should be the same across all samples to ensure equal stretch conditions between the samples.

4. Remove wrinkles in the electrospun scaffold by gently pulling on the electrospun scaffold. Again, make two knots at both sides with a suture wire on the upper end of the scaffold at the location of the upper engraved groove on the pressure conduit.

5. Release the scissor clamp, and cut away the excess of silicone tubing with a knife, leaving $20-30 \%$ of the screw thread covered with silicone tubing, to prevent leakage when the nose cone is mounted on the screw thread.

NOTE: Repeat steps 2.4 and 2.5 for all dynamic samples.

6. For the static control samples, secure the electrospun scaffold mounted on the silicone tubing on pressure conduits without holes. These conduits can be kept separately in a $15 \mathrm{~mL}$ tube until seeding (step 4) and do not need to be mounted in the flow culture chamber compartments.

6. Decontaminate the partly constructed flow culture chambers with electrospun scaffold by exposing it to UV light for $10 \mathrm{~min}$. Turn the flow culture chambers with electrospun scaffolds to the other side, and repeat UV light exposure for $10 \mathrm{~min}$.

7. Screw the nose cones on the screw thread of the pressure conduits with holes for the dynamic samples.

1. Make sure the top end of the silicone tubing fits into the nose cone to prevent leakage in later stages. If there is too much silicone tubing, cut the excess of tubing away with a knife.

2. Place the partly constructed flow culture chambers in a large Petri dish, and point the nose cone towards the UV light source. Apply UV illumination for $5 \mathrm{~min}$.

8. Complete construction of the flow culture chamber with the glass tube and top compartment of the flow culture chamber (Figure 1A-B).

1. Pre-wet the electrospun scaffolds by dipping the pressure conduit with the silicone tubing and electrospun scaffold in $30 \%$ ethanol, followed by a dip in ultrapure water two times.

2. Place the glass tube over the pressure conduit, and push gently in the bottom compartment and gently secure it.

3. Take the top compartment containing the flow inlet, place a silicone O-ring, the flow straightener, and the adapter bushing in the correct order (Figure 1A-B), and place over the open end of the glass tube and gently secure it. 
4. Screw a white Luer cap on the flow inlet of the top compartment.

5. Remove the male Luer plug from the flow outlet of the bottom compartment, and clean the surface around it with an ethanol-soaked paper tissue.

6. Place a syringe with $10 \mathrm{~mL}$ of ultrapure water in the flow outlet, open the white Luer cap on the top compartment, and fill the chamber with ultrapure water. Close the white Luer cap again, remove the syringe, clean again with ethanol, and close the flow outlet with a male Luer plug.

NOTE: Repeat steps 2.6-2.8 for all flow culture chambers.

7. For the static controls, add $10 \mathrm{~mL}$ of ultrapure water to the $15 \mathrm{~mL}$ tubes holding the samples mounted on the pressure conduits without holes.

9. Place all flow culture chambers in the incubator. Replace the ultrapure water with culture medium one day before cell seeding on the same way as described in steps 2.8 .5 and 2.8.6 (make sure to collect the 'old' ultrapure water with an ethanol-soaked paper tissue placed directly on the flow outlet).

[The protocol can be paused here]

\section{Preparations for the flow pump setup}

NOTE: Perform step 3 in a laminar flow cabinet.

1. Collect all pump setup materials and prepare for usage. NOTE: Experimenters are referred to the manufacturer's protocol for a detailed description of setting up the pump, the fluidic units, and medium tubing through the valves of the fluidic unit.

1. Set the pump to 200 mbar capacity.
2. Screw the reservoir holders for $60 \mathrm{~mL}$ reservoirs to the fluidic units.

3. Clean the re-usable rubber air filters with a paper tissue soaked in ethanol, make sure the air filter stays dry.

2. Place the $60 \mathrm{~mL}$ reservoirs in the reservoir holders, and place the standard medium tubing through the valves of the fluidic unit. Connect the medium tubing with a larger inner diameter with female Luer lock couplers into an enclosed loop.

3. Clamp the medium tubing with a hose clip, directly below the reservoirs.

4. Fill the reservoirs with $25 \mathrm{~mL}$ of culture medium per 60 $\mathrm{mL}$ reservoir. Release the hose clip, and let the medium enter the tubing.

5. Close the medium reservoirs with the rubber air filters, and place the flow pump setup in the incubator till step 4 .

\section{Cell Seeding Using Fibrin as a Cell Carrier}

NOTE: Perform step 4 in a laminar flow cabinet.

1. Prepare the fibrin gel for the cell seeding step. For details, see Mol et al. ${ }^{67}$ For the fibrin gel, the fibrinogen solution should have a final concentration of $10 \mathrm{mg} / \mathrm{mL}$ (correct for the purity of the protein stock), and the thrombin solution should have a final concentration of $10 \mathrm{U} / \mathrm{mL}$.

1. Thaw fibrinogen to RT, before weighing $\sim 50 \mathrm{mg}$ (enough for 10 samples) in a plastic container with a red lid.

2. Add cell culture medium to prepare the fibrinogen solution (at a concentration of $10 \mathrm{mg} / \mathrm{mL}$, correct for the purity of the protein stock). Mix well and filter to sterilize the fibrinogen solution with a $0.2 \mu \mathrm{m}$ syringe 
filter into a sterile $15 \mathrm{~mL}$ tube. Keep the filtered fibrinogen solution on ice.

NOTE: Avoid preparing the fibrinogen solution too long in advance, otherwise the fibrinogen may clot spontaneously.

3. Thaw thrombin and make a thrombin solution (at a concentration of $10 \mathrm{U} / \mathrm{mL}$ ) in cell culture medium and place on ice. Prepare $20 \mu \mathrm{L}$ thrombin + cells solution per sample. For $n=10$ samples, $200 \mu \mathrm{L}$ is needed; therefore, prepare $250 \mu \mathrm{L}$ thrombin solution to account for possible pipetting errors.

2. Collect and count the cells from the culture flasks. Mix the cells in the desired ratio and amount $\left(1.2 \times 10^{6}\right.$ monocytes and $0.6 \times 10^{6}$ myofibroblasts per scaffold). Make sure there are enough cells for $n+1$ samples to correct for pipetting errors. Centrifuge at $350 \times g$ for 10 min at RT. Remove the supernatant.

3. Make a mixture of the suspended cells and thrombin.

1. For each sample, use $20 \mu \mathrm{L}$ of the thrombin solution. For $\mathrm{n}=10$ samples, add $200 \mu \mathrm{L}$ thrombin to the cell pellet and mix. Measure the volume of the cell suspension (cells + thrombin), and calculate how to divide evenly over all 10 scaffolds (e.g., if the thrombin + cell suspension has a volume of 260 $\mu \mathrm{L}$, each electrospun sample will receive $260 \mu \mathrm{L} / 10$ samples $=26 \mu \mathrm{L}$ thrombin + cell suspension) .

2. As the seeding of the scaffolds is performed in two steps, prepare two $1.5 \mathrm{~mL}$ microfuge tubes that will hold half of the cell suspension for each scaffold (in the example calculation of the previous step: prepare two tubes with $13 \mu \mathrm{L}$ of thrombin + cell suspension). Place on ice.
NOTE: The following steps, especially step 4.4 , are ideally performed by two experimenters.

4. Dry the pre-wetted electrospun scaffolds with vacuum to prepare for cell seeding.

1. Connect a glass Pasteur pipet to the vacuum system of the laminar flow cabinet, and place in an empty 50 $\mathrm{mL}$ tube for sterile temporary storage.

2. Take the flow culture chambers from the incubator, remove the male Luer plug from the flow outlet, and remove the medium after opening the white Luer cap and placing an ethanol-soaked paper tissue in front of the flow outlet.

3. Take off the top compartment and the glass tube, and place in a sterile Petri dish for temporary storage.

4. Place the vacuum Pasteur pipet on the electrospun scaffold, and remove as much medium as possible. CAUTION: Vacuum dry the electrospun scaffold very gently. Instead of a back-and forth linear motion over the scaffold, place the vacuum pipet at multiple locations. Clamp the vacuum tubing on top of the Pasteur's pipet in between the fingers for better control.

5. Mix the fibrinogen solution in a 1:1 ratio with the thrombin + cell suspension (e.g., mix $13 \mu \mathrm{L}$ of fibrinogen with $13 \mu \mathrm{L}$ of thrombin + cell suspension). To make sure that the fibrin polymerizes in the scaffold and not in the microfuge tube, pipet the fibrinogen, turn the pipet wheel for the 'extra volume' of the thrombin + cell suspension, and pipet up and down once in the microfuge tube with cell suspension to mix. 
6. Directly homogeneously drip the solution over the full length of the electrospun scaffold. It is advised that Experimenter $A$ drips the fibrin mixture, while experimenter B holds the bottom compartment with the electrospun scaffold mounted to the pressure conduit.

7. After the fibrin with the cells is dripped over the electrospun scaffold, Experimenter B slowly moves the scaffold, from left to right and up and down, to further divide the cells evenly over the scaffold.

8. Repeat step 4.4.5 - 4.4.7 on the other side of the electrospun scaffold.

9. Mount the flow culture chamber again by carefully placing the glass tube (prevent fibrin sticking and clotting to the inner side of the glass tube), and push back the top compartment of the flow culture chamber. Directly place the seeded construct without any medium or phosphate-buffered saline (PBS) in the flow culture chamber in the incubator.

10. Repeat steps 4.4.1-4.4.9 for all dynamic samples. For the static samples mounted to pressure conduits without holes, seed according to steps 4.4.1-4.4.8, and place in a $15 \mathrm{~mL}$ tube afterwards.

11. Let the fibrin polymerize for $60 \mathrm{~min}$ in the incubator. [The protocol can be paused here for 30-60 min.]

5. After polymerization, fill the flow culture chambers (dynamic samples) or the $15 \mathrm{~mL}$ tubes (static samples) with medium.

\section{Coupling of the bioreactor and flow pump systems before starting experiment}

NOTE: Perform steps 5.1-5.3 in a laminar flow cabinet.
1. Take the tray carrying the flow culture chambers and the fluidic units with filled medium reservoirs and connected medium tubing inside the laminar flow cabinets.

2. Position the flow culture chambers on the bioreactor base for the experimental groups loaded with cyclic stretch and with combined hemodynamic loads (Figure 1E).

1. Tilt the flow culture chamber upside down, and fill the pressure conduit from below with ultrapure water using a syringe with thin tubing (this can be of any type, as long as it is flexible and thin, in this experiment, a $10 \mathrm{~cm}$ long, $0.15 \mathrm{~mm}$ inner diameter wire was attached to the needle).

2. Place the thin tubing inside the pressure conduit, and while the pressure conduit is filled with ultrapure water by gradually pushing the water out of the syringe, pull the wire out of the pressure conduit simultaneously, to make sure that there are no air bubbles inside the pressure conduit.

3. Place the flow culture chamber on one of the eight screw threads on bioreactor base. Place a silicone O-ring between the bioreactor base and the white Luer connector to prevent possible leakage, and tighten the white Luer connector from the bottom compartment.

4. Repeat steps 5.2 .2 and 5.2 .3 for all cyclically stretched samples.

3. Connect the flow culture chambers for all experimental groups, except the static control, to the flow pump system.

1. Place a hose clip on the medium tubing. Remove the white Luer cap covering the flow inlet of the top compartment of the flow culture chamber. Remove 
the female Luer coupler of the medium tubing, and connect the medium tubing on one side with the flow inlet on the top compartment, and the other side of the medium tubing with flow outlet at the bottom compartment.

2. Repeat step 5.3.1 for all flow culture chambers. At this point, the bioreactor and the flow culture chambers are filled with medium and connected to the flow systems.

3. For the static control samples, place the samples vertically in a cell culture flask with filter cap by using the scissor clamp. Fill the cell culture flask with medium, and place in the incubator.

4. Transfer the complete setup from the laminar flow cabinet to the incubator, and connect the fluidic units to the air pressure tubing and the electric cable.

5. Start the software, and initialize the flow pumps. Start the medium flow for the samples one by one.

1. Check if the valves of the fluidic unit are clicking.

2. Remove the hose clamp from the medium tubing.

3. Start the flow pump with $100 \mathrm{mbar}$ and $10 \mathrm{~s}$ switching time.

4. Carefully check the flow direction for possible leakage or air bubbles. Any entrapped air bubbles can be removed by turning the flow culture chamber upside down

NOTE: Make sure that the medium levels in the medium reservoirs are balanced, to prevent suction of air into the system and air bubbles in the flow culture chambers, and to not allow the reservoirs to run dry (Figure 2C).

5. Repeat step 5.5 for all fluidic units one-by-one.
6. Initialize the strain pump.

1. Connect the pneumatic actuated pump via the air inlet on the pneumatic cylinder to the compressed air. Connect the lower air outlet with the blue tubing for air out (Figure 1F).

2. Open LabVIEW software, run the LabVIEW script and compressed air pressure application system, as described by Van Kelle et al. ${ }^{68}$, enter displacement and frequency (start with low frequency of $0.2 \mathrm{~Hz}$ ). Pause the pump when the Teflon bellow is at its lowest level.

3. Place the pressure sensor in the pressure sensor inlet on the hydraulic reservoir.

7. Change the pump settings to the desired settings (for 1.5 $\mathrm{Pa}$, use 150 mbar, $10 \mathrm{~s}$ switching time).

8. Start the strain pump, and apply the preferred setting (e.g., $0.5 \mathrm{~Hz}, 1.05$ stretch).

\section{Running Experiment for Multiple days; Monitoring of Shear and Stretch During Culture and Medium Replacement}

1. Calculate the WSS at the scaffold wall.

1. Record the flow magnitude every other day (see the flow pump manufacturer's manual for details). In short, observe the change in liquid levels (in $\mathrm{mL}$ ) in the medium reservoirs in between the switching of the fluidic unit reservoir for $10 \mathrm{~s}$. Conduct at least five measurements, calculate the mean value, and multiply by 6 to get the flow rate $Q$ in $\mathrm{mL} / \mathrm{min}$.

2. The flow is described by a Poiseuille flow through an annular channel. Assuming culture medium as a Newtonian fluid, calculate the WSS at the scaffold wall, $\mathrm{r}_{1}$, by Equation 1 . 
$\tau_{w}=\frac{1}{4}\left(\frac{-\partial p}{\partial z}\right)\left[\frac{1}{r_{1} \ln \left(\frac{r_{2}}{r_{1}}\right)} *\left(r_{2}^{2}-r_{1}^{2}\right)-2 r_{1}\right]$

where the WSS $T_{W}$ at the scaffold wall ( $r_{1}$; here $r_{1}$ $=1.7 \mathrm{~mm}$ ), resulting from a steady state flow, is determined by the applied pressure $p$ and the inner radius of the glass tube $r_{2}$ (here $r_{2}=2.3 \mathrm{~mm}$ ). The pressure gradient in the axial direction is assumed to be uniform between the flow inlet and flow outlet and is given by Equation 2 (Figure 1J).

$\frac{-\partial p}{\partial z}=\frac{8 \mu Q}{\pi\left(r_{2}^{2}-r_{1}^{2}\right)}\left[r_{2}^{2}+r_{1}^{2}-\frac{r_{2}^{2}-r_{1}^{2}}{\ln \left(\frac{r_{2}}{r_{1}}\right)}\right]^{-1}$

with $\mu$ the dynamic viscosity (here medium viscosity was assumed constant, $\mu=0.7 \times 10^{-3} \mathrm{~Pa} \cdot \mathrm{s}$ at $37^{\circ} \mathrm{C}$ ) and $Q$ the applied flow rate.

2. Monitor the stretch applied to the scaffolds every other day.

1. Place a dark background behind the flow culture chamber to increase the contrast between the scaffold and the background. Position the LED light lamps, pointing towards the scaffold, to help the visualization of the scaffold.

2. Take time-lapse photographs of the scaffold at a frequency of $30 \mathrm{~Hz}$ for $6 \mathrm{~s}$ (i.e., 3 stretch cycles) with a high-speed camera.

NOTE: A lower recording frequency may suffice if the camera permits. However, the minimally required frequency was not determined.

3. Manually determine the minimum and maximum diameter of the scaffold from the images.

4. Calculate the minimum and maximum outer diameter of the electrospun scaffold to calculate the maximum stretches according to Equation 3. $\lambda_{\theta}=\frac{d_{1}}{d_{0}}(3)$

where the circumferential stretch $\left(\lambda_{\theta}\right)$ is given by the ratio between the outer diameter of the scaffold, $d_{1}$, and its initial diameter, $d_{0}$.

3. Correct for medium evaporation, and refresh medium three times per week.

1. Stop and decouple the cables for the flow systems and the strain pump.

2. Place hose clips on the medium tubing.

3. Determine how much medium evaporated based on the volume indicator marks on the medium reservoirs.

4. Transfer the tray with the bioreactor and the fluidic units to the laminar flow cabinet.

5. Remove the rubber air filters of the medium reservoirs; add autoclaved ultrapure water to compensate for the evaporated volume of medium. Close the medium reservoirs again, and connect to the pump again to mix the medium with the ultrapure water.

6. Repeat steps 6.3.1-6.3.5. Remove the rubber air filters again, take out $25 \mathrm{~mL}$ of culture medium, and spin down at $300 \times g$ for $5 \mathrm{~min}$ at RT.

1. Collect $1.5 \mathrm{~mL}$ supernatant, and store at $-30{ }^{\circ} \mathrm{C}$ for analysis of secretory profiles (for analysis with enzyme-linked immunosorbent assay (ELISA)).

2. Collect the desired volume of supernatant for paracrine signaling studies, by using the supernatant as conditioned medium ${ }^{43}$. 
7. Add $25 \mathrm{~mL}$ of fresh medium to the medium reservoirs.

8. Place rubber air filters back on the medium reservoirs.

9. Place the complete setup back in the incubator; connect all cables and air tubing to the pump and strain pump. Release the hose clips and repeat steps $5.4-5.8$.

4. Check if silica drying beads in the drying bottles connected to the pump are moist (white appearance), and replace with dry silica beads if required (orange appearance).

\section{Ending Experiment, Sample Collection, and Equipment Cleaning and Storage}

1. On the last day of the experiment, correct for medium evaporation as described in steps 6.3.1-6.3.5, and harvest the samples one-by-one.

1. To harvest the samples one-by-one, the flow pump and strain pump need to be paused several times. Place a hose clip on medium tubing. Temporarily stop the flow pump and the strain pump. Disconnect one flow culture chamber from the bioreactor base; replace by a white Luer cap on the bioreactor base. Take the flow culture chamber and fluidic unit to the laminar flow cabinet. Start the flow pump and strain pump again to apply the hemodynamic load to the other samples until harvesting.

2. Collect medium from the medium reservoirs for paracrine cytokine production analysis via ELISA.

2. Decouple flow units and harvest tubular construct. Section according to the desired cutting scheme. Parts of the construct can be stored at $4{ }^{\circ} \mathrm{C}$ (after $15 \mathrm{~min}$ fixation in $3.7 \%$ formaldehyde and $3 \times 5$ min washing in PBS) or $-30{ }^{\circ} \mathrm{C}$ (after snap-freezing in liquid nitrogen) until further analysis.

3. Clean the bioreactor and pump components. Additionally, the advised cleaning method per item is mentioned in the Table of Materials.

1. Clean the rubber air filters with $70 \%$ ethanol. Be very careful to not moisten the inner filter!

2. Collect all separate components: medium tubing, medium reservoirs, glass tubes, male Luer plugs and female Luer locks, white Luer caps, pressure conduits, nose cones, silicone O-rings, adapter bushing, flow straighteners (excluding pumps, fluidic units, rubber air filters, the bioreactor base), and rinse in running tap water.

3. Place $\mathrm{O} / \mathrm{N}$ in $0.1 \%$ sodium dodecylsulfate in deionized water.

NOTE: Do not use ultrapure water as the parts might rust.

4. Rinse with tap water and dishwashing soap.

5. Immerse in deionized water, followed by $70 \%$ ethanol two times, followed by deionized water.

6. Place all materials separately on paper tissues and let them dry. Use pressured air to dry tubing.

7. Clean all non-autoclavable materials with a paper tissue soaked in $70 \%$ ethanol. This includes the rubber air filter (bear in mind that the air filter should stay dry) and the bioreactor base (Teflon bellow and pneumatic cylinder).

8. Autoclave the components of the fluidic chamber (including the silicone O-ring), the medium tubing, the medium reservoirs (without the rubber air filter), 
male Luer plugs and female Luer couplers, white Luer caps, hose clips, and standard equipment (e.g., tweezers, clamping scissors)

9. For convenient use during next experiments, combine the separate components for one complete fluidic chamber in an autoclavable box.

4. Remove water from the hydraulic reservoir. Clean with $70 \%$ ethanol, followed by deionized water. Let it dry. Refill with deionized water and a few drops of water-bathpreserving disinfectant.

5. Store the glass tubes for the flow culture chamber in $70 \%$ ethanol.

6. Place the moist silica drying beads (white appearance) in the oven $\mathrm{O} / \mathrm{N}$ at $120{ }^{\circ} \mathrm{C}$ to let them dry (orange appearance), and store in an air-tight flask.

\section{Representative Results}

This bioreactor was developed to study the individual and combined effects of shear stress and cyclic stretch on vascular tissue growth and remodeling in 3D biomaterial scaffolds. The design of the bioreactor allows for culturing up to eight vascular constructs under various loading conditions (Figure 1A). The vascular constructs are positioned in a flow culture chamber (Figure 1B) in which both the circumferential stretch and WSS can be independently controlled. The top compartment of the flow culture chamber holds a flow straightener to stabilize the flow in a relatively short settling length (Figure 1C). Directly downstream of the flow straightener, the nose cone distributes the flow evenly through the annular channel (Figure 1D). When all steps of the protocol are performed in the correct manner, the vascular scaffolds in the flow culture chamber can be subjected to a continuous unidirectional flow through the annular channel between the scaffold and the glass wall and are circumferentially stretched by the pneumatic pump (Figure $1 \mathrm{E}-\mathrm{F})$. Before the scaffold is mounted, the electrospun tube should be cut into $25 \mathrm{~mm}$ tubes (Figure 1G) and can be examined with SEM to analyze the microarchitecture (Figure 1H-I). It is important to note that the PCL-BU grafts in this example can be replaced by any other elastomeric tissue engineered grafts (natural or synthetic origin, different microarchitecture or porosity). The inside-out design allows testing of highly porous grafts as it does not necessarily have to be leak-free. The schematic image of the flow culture chamber shows the physical interpretation of the parameters used in the equations to describe the WSS (equation 1), the pressure gradient (equation 2), and the circumferential stretch (equation 3) (Figure 1J).

Incorrect execution of the critical steps of the protocol can result in a few scenarios. For example, leakage from the hydraulic reservoir can occur as a result of incorrectly mounted knots, leading to leakage of hydraulic fluid from the pressure conduits with holes passing the silicone tube and entering the flow culture chamber (Figure 2A). Leakage of the hydraulic fluid at the connection between the screw threads and the bioreactor base can also occur when the silicone ring is not well placed or if the Teflon bellow was not allowed to slightly expand $\mathrm{O} / \mathrm{N}$ one day before the experiment (Figure 2B). Furthermore, when medium is not degassed, or if the medium levels in the medium reservoirs are not well balanced and one medium reservoir runs dry, resulting in air being sucked into the system, air bubbles can arise in the flow culture chamber (Figure 2C), which disturbs the WSS patters, compromising cell viability and subsequent tissue growth. Lastly, when the silicone O-ring in the bottom compartment 
is not correctly placed, medium spill may be observed below the flow culture chamber (Figure 2D).

As this bioreactor setup allows for the application of individual and combined hemodynamic loads, multiple hemodynamically loaded experimental groups can be included in one experiment (Figure 3A). Previously, different hemodynamic loads (i.e., two shear stress regimes and two stretch regimes) were validated by applying various possible system settings (Figure 3B). When stretch (Figure 3C) and WSS (Figure 3D) were monitored over long-term culture periods, it was validated that these can be maintained at relatively constant levels over a period of up to 20 days.

The bioreactor is especially well suited to study the influence of hemodynamic loading on growth and remodeling in an in situ vascular TE context. The stages of in situ TE are hypothesized to mirror the stages of the natural wound healing response (Figure $4 \mathrm{~A}$ ). The co-cultures of monocyte-derived macrophages and myofibroblasts derived from human saphenous veins, as described here, were established as an in vitro mimic of the proliferative phase. Three days after seeding, immunofluorescence staining showed a homogenous distribution of both cell types throughout the scaffold (Figure 4B). After 20 days of coculture, cyclic stretch alone resulted in the deposition of more numerous and thicker collagen type I fibers, while in the group with combined hemodynamic loads, this effect of cyclic stretch was overruled by shear stress, resulting in less pronounced collagen type I deposition, illustrated here by immunofluorescence staining (Figure 4C). For successful in situ tissue regeneration, a tight balance between tissue production and scaffold resorption is required. In addition to tissue formation, the bioreactor allows for the induction of cell-driven scaffold resorption. For example, when culturing a mono-culture of macrophages for 8 days on the electrospun grafts, fiber erosion and fiber cleavage were observed in all hemodynamic loading regimes, with the most pronounced resorption in the static group and least pronounced resorption in the shear stress group (Figure 4D). Together, these results show the impact of the different hemodynamic loading regimes on both growth and remodeling. These insights are helpful in optimizing the design parameters for newly developed in situ TEVGs.

Another important determinant of the tissue regeneration process is the presence of pro- and anti-inflammatory cytokines. As the bioreactor is a closed-loop system, the cells in the system will be continuously exposed to the paracrine stimuli of secreted factors. The cytokine secretion profiles in the medium of the dynamically loaded co-cultures of human peripheral blood mononuclear cell (PBMC)-derived macrophages and human myofibroblasts from saphenous veins were analyzed in early and later stages (Figure 5A). These representative results illustrate the impact of both cyclic stretch and shear stress on the cytokine secretion profile in the co-culture setup. Interestingly, the combined effects of both loads showed either dominance of one of the two loads (e.g., cyclic stretch for interleukin-6 (IL-6) and monocyte chemoattractant protein-1 (MCP-1)) or synergistic effects of both loads (e.g., for IL-10) (Figure 5B). These insights, gathered by using this in vitro testing platform, give valuable information for the development of in situ TEVGs that are based on the rationale of macrophage-driven in situ tissue regeneration.

Co-culture experiments of macrophages and myofibroblast showed that the mechanical environment and the resulting loading-dependent inflammatory environments modulated the phenotype of the myofibroblasts. After 20 days of 
hemodynamic loading, gene expression of myofibroblast markers showed clear differences in the individual and combined impact of the loading and in direct and paracrine signaling of macrophages on myofibroblasts (Figure 6A). Furthermore, the gene expression patterns of contractile marker alpha smooth muscle actin correlated with protein synthesis (Figure 6B). Furthermore, cyclic stretch stimulated collagenous and elastic matrix gene expression and attenuated matrix metalloproteinase $1 /$ tissue inhibitor matrix metalloproteinase 1-mediated collagen remodeling, whereas a stabilizing effect of shear stress was observed in the coculture (Figure 6C). These long-term co-culture experiments show the possibility of studying the later stage tissue remodeling phase (Figure 4A) in various hemodynamic loading regimes in tissue engineered vascular grafts with this bioreactor. As the TEVG are mounted "inside out" on a silicone tube, the circular stretch and WSS can be applied for longer culture periods. 

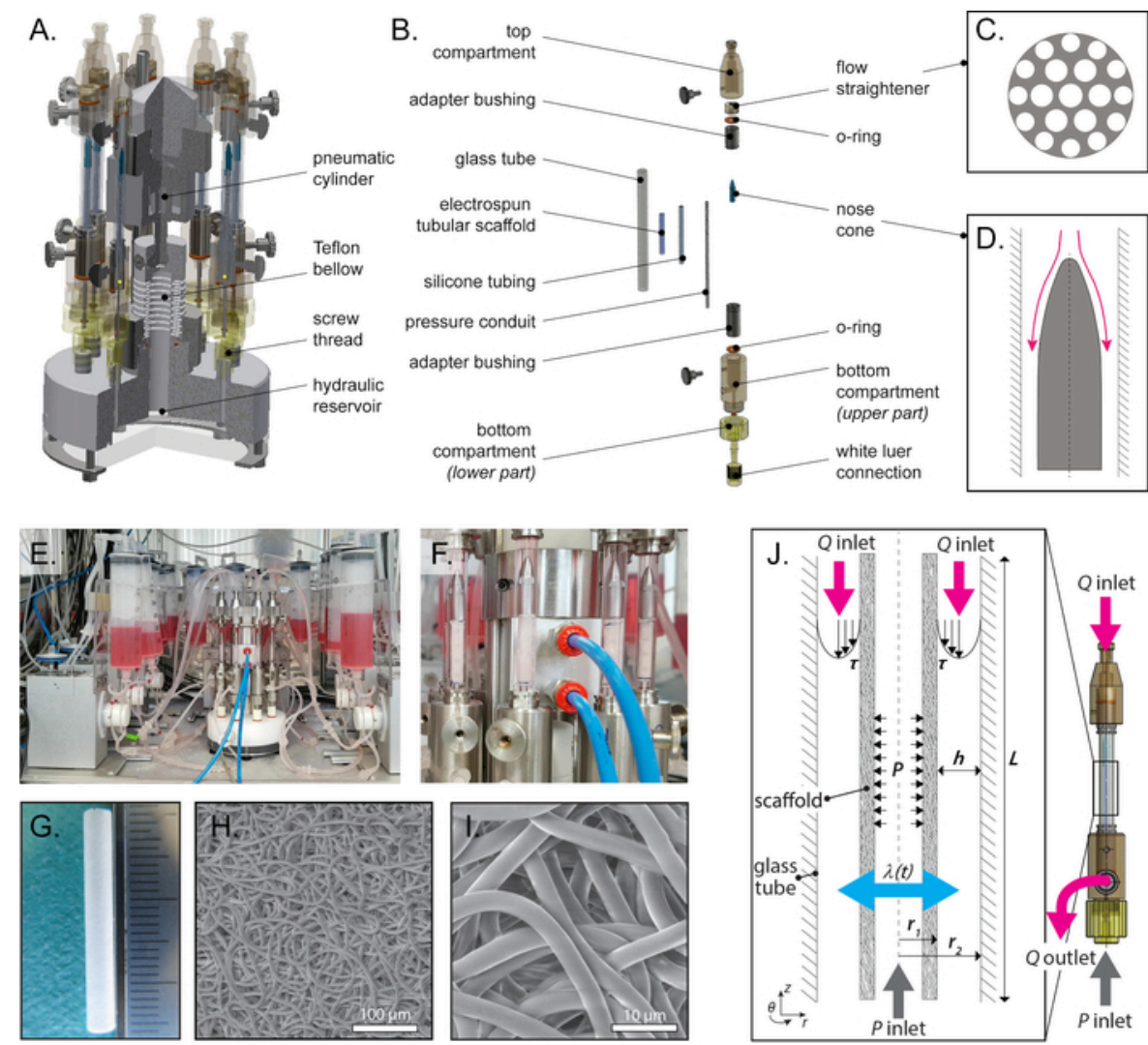

Figure 1: Design and overview of the bioreactor. (A) Construction drawing of the bioreactor base and (B) exploded view of the fluid culture chamber with all parts indicated (steps 2.4-2.8). The top compartment of the flow culture chamber holds a flow straightener. (C) The spherically blunted nose cone is positioned after the flow straightener to distribute the flow through the annular channel (flow direction indicated in pink). (D) Together, these components control and guide flow directionality. See Table 1 for a functional description of the individually mentioned parts. (E) Photograph of complete bioreactor setup (step 5.2) and (F) a close-up view of the flow culture chambers and pneumatic cylinder (step 5.6.1). (G) Gross appearance of the electrospun PCL-BU scaffold before seeding (ruler ticks $1 \mathrm{~mm}$ ). Scanning electron microscopic images of tubular electrospun PCL-BU scaffold with $3 \mathrm{~mm}$ inner diameter and $5 \mu \mathrm{m}$ average fiber diameter at different magnifications, scale bars (H) $100 \mu \mathrm{m}$ and (I) $10 \mu \mathrm{m}$ (step 1.3). (J) Schematic image of the culture chamber consisting of a tubular electrospun scaffold, with outer radius $\left(r_{1}\right)$ centered in a glass tube of inner radius $\left(r_{2}\right)$. The flow $(Q)$ inlets and outlets are connected to the annular ring, with channel height $h$ for applying wall shear stress $(t)$. The pressure/stretch $(P)$ inlet is connected to the silicone-mounted scaffold for applying a circumferential stretch $(\lambda(t))$ to the mounted electrospun scaffolds from the inside (step 6.1). Abbreviations: polycaprolactone bisurea (PCL-BU). Panels C, D and G-J were adapted from Van Haaften et al. ${ }^{19}$. Please click here to view a larger version of this figure. 


\begin{tabular}{|c|c|}
\hline Bioreactor parts & Functional description \\
\hline \multicolumn{2}{|c|}{ Stretch application } \\
\hline Pneumatic cylinder & Actuates Teflon bellow. \\
\hline Teflon bellow & Loads the hydraulic reservoir. \\
\hline Hydraulic reservoir & $\begin{array}{l}\text { Can be connected with up to } 8 \text { flow culture } \\
\text { chambers, is filled with demi water, applies } \\
\text { pressure to the silicone mounted constructs. }\end{array}$ \\
\hline Screw thread & $\begin{array}{l}\text { Connection between the flow culture chamber } \\
\text { and hydraulic reservoir. The pressure } \\
\text { inlet for the silicone mounted constructs. }\end{array}$ \\
\hline White luer connector & $\begin{array}{l}\text { Used to screw the flow culture chamber } \\
\text { tight to one of the eight screw threads on } \\
\text { the hydraulic reservoir/bioreactor base. }\end{array}$ \\
\hline Pressure conduit with small holes & $\begin{array}{l}\text { Directly connected with water in hydraulic reservoir. } \\
\text { When the hydraulic reservoir is pressurized, the pressure } \\
\text { conduits fill the space in between the silicone tube (that } \\
\text { is mounted on the pressure conduit) with water, pushing } \\
\text { the silicone tubing outwards, resulting in a circumferential } \\
\text { stretch on the silicone mounted graft from inside. }\end{array}$ \\
\hline Silicone tubing & $\begin{array}{l}\text { To mount the electrospun graft on the pressure conduit. The } \\
\text { silicone tubing is circumferentially stretched from inside. }\end{array}$ \\
\hline \multicolumn{2}{|c|}{ Flow application } \\
\hline Flow pump system & $\begin{array}{l}\text { Used to control the flow in the flow culture chambers. } \\
\text { (in our example: an ibidi pump system is used.) }\end{array}$ \\
\hline Top and bottom compartment with flow inlet and outlet & Connects the flow culture chamber into the flow loop. \\
\hline Glass tube & $\begin{array}{l}\text { Contains the pressurized scaffold in the } \\
\text { center and allows perfusion of the scaffolds. }\end{array}$ \\
\hline Flow straightener & Stabilizes the flow in a relatively short settling length. \\
\hline Nose cone & Distributes the flow evenly. \\
\hline Adapter bushing & Fixes the glass tube. \\
\hline
\end{tabular}


Table 1: Functional description of bioreactor main features, corresponds with indicated parts in Figure 1A-D.
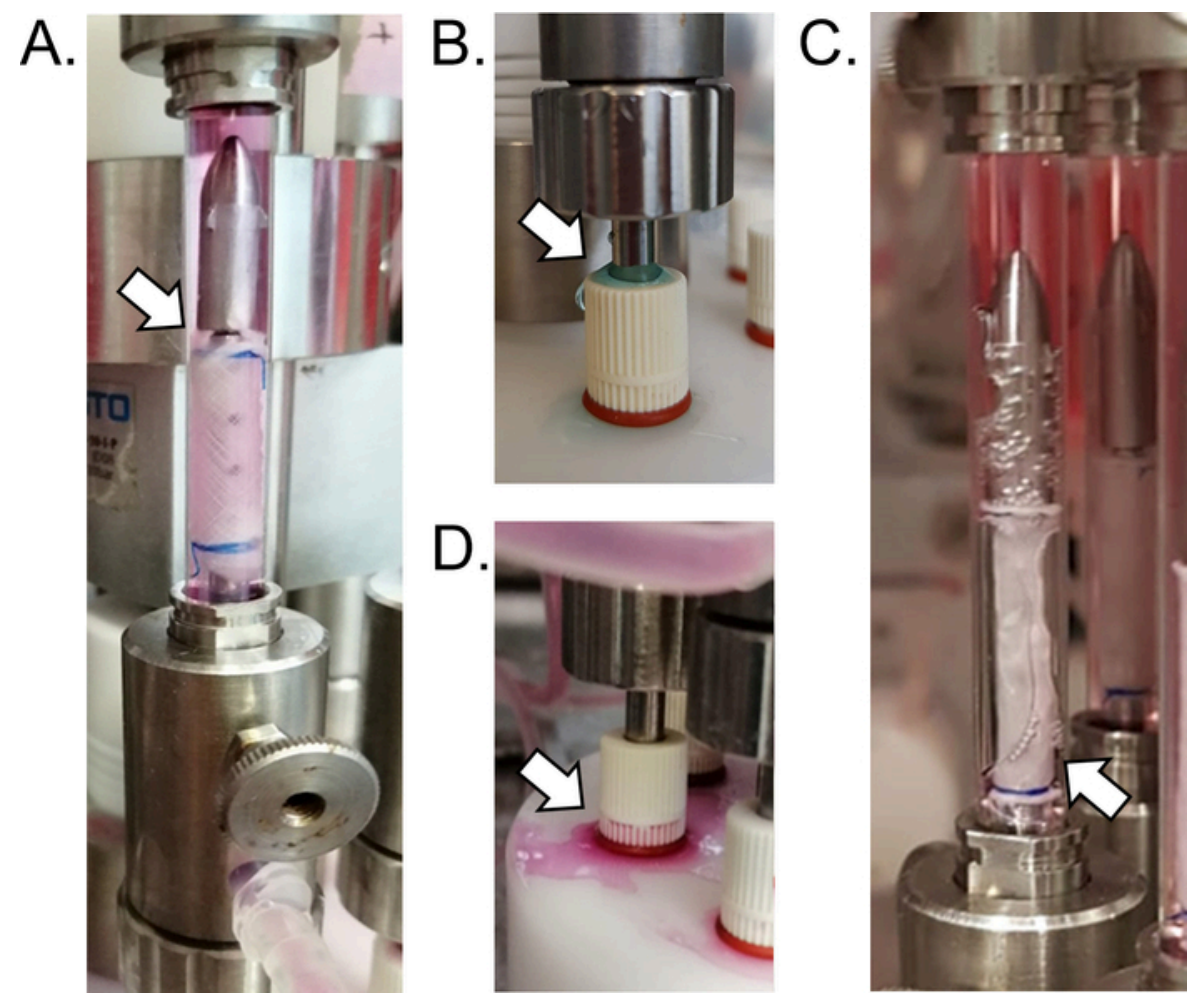

Figure 2: Results of inaccurate execution of the critical steps in the protocol. Photographs of some occurrences that can be observed in the bioreactor setup when critical steps are not performed in the correct manner. (A) If the knot is not tight enough or not exactly placed in the engraved groove (indicated by arrow), a slight leakage of hydraulic fluid into the flow culture chamber may occur (step 2.5). (B) If the Teflon bellow was not allowed to expand O/N one day before the experiment or when the silicone ring is not well placed, hydraulic liquid leakage from the hydraulic liquid at the connection between the screw threads and the bioreactor base might occur (indicated by arrow) (step 1.4 and 5.2.3). (C) Air bubbles in the flow culture chamber (indicated by arrow) will result in disturbed shear stress patterns. Always degas the culture medium and make sure that the medium levels in the medium reservoirs are balanced to prevent one reservoir running dry and air getting sucked into the flow chamber system (steps 1.2 and 5.5.4). (D) When the silicone ring in the bottom compartment is not placed correctly, spillage of the medium may be observed (indicated by arrow) (step 2.4.2). Please click here to view a larger version of this figure. 
A.

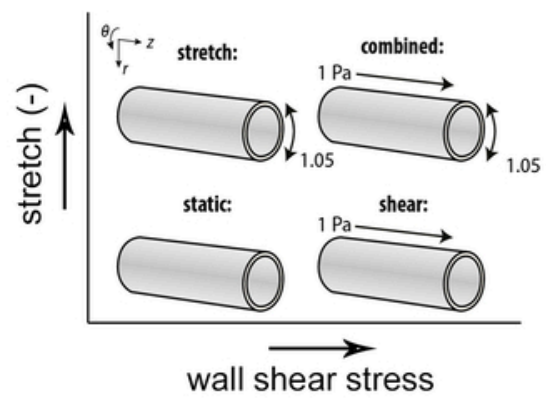

C.

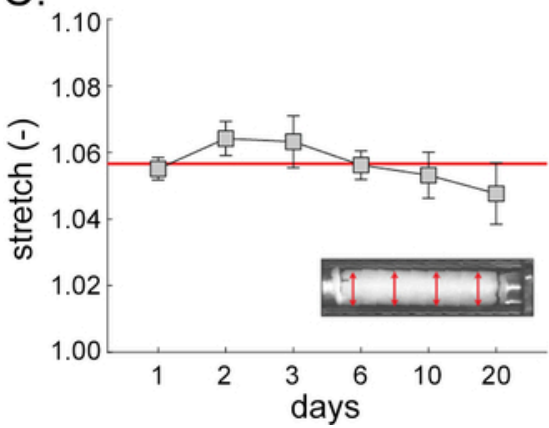

B.

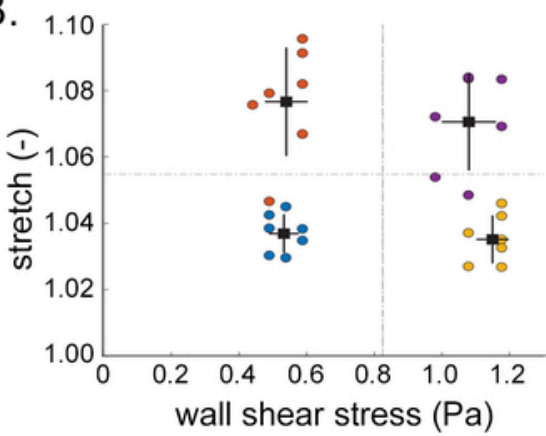

D.

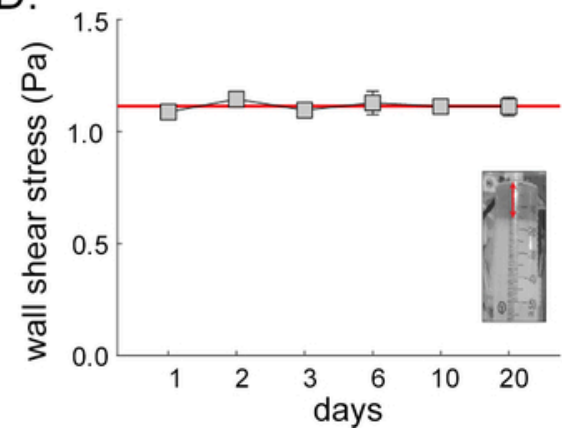

Figure 3: Control of shear stress and stretch. As the bioreactor allows for independent and combined application of stretch and shear, (A) multiple experimental groups can be included in one experiment. (B) Examples of variations in maximum stretches and shear stresses at a specific point in time tested under four distinct system settings (indicated by the colors). Black rectangles represent mean \pm standard deviation of the measurements for each setting. The dotted lines are computed as the mean of the stretches (horizontal line) and the shear stresses (vertical line) to indicate the four distinct loading conditions. (C) Cyclic circumferential stretches in the cyclic stretch and combined groups over the course of the experiment of 20 days, based on outer diameter measurements of the scaffold constructs monitored with a time lapse of high-speed camera (step 6.2). (D) Monitored wall shear stresses in the shear stress and combined groups over the course of the experiment, based on the changing medium levels in the syringe (step 6.1). Panels A, C, and D were adapted from Van Haaften and Wissing et al. ${ }^{44}$; panel B was adapted from Van Haaften et al. ${ }^{19}$. Please click here to view a larger version of this figure. 
A.

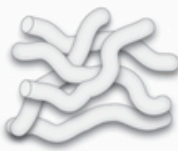

implantation

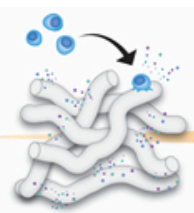

inflammation
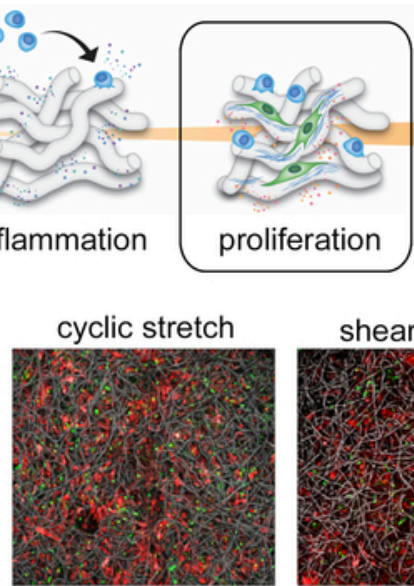

C.

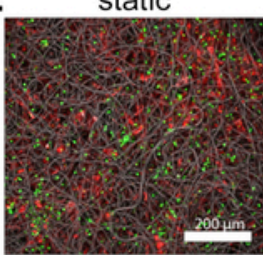

static

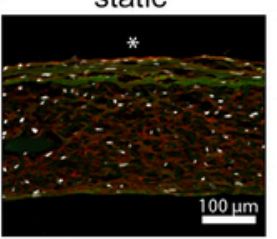

D.

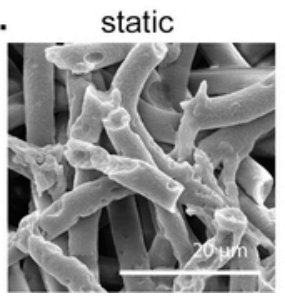

cyclic stretch

cyclic stretch

shear stress

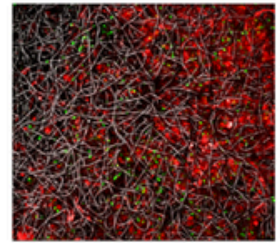

scaffold macrophage HVSC

shear stress

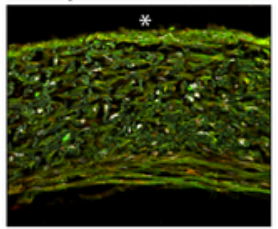

cyclic stretch

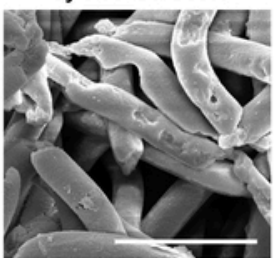

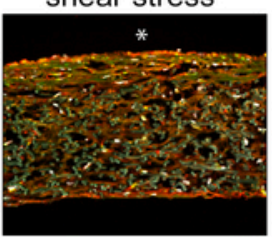

Collagen I Collagen III

shear stress

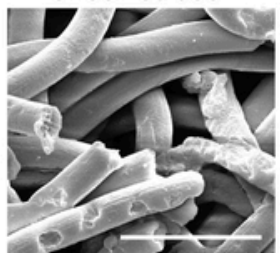

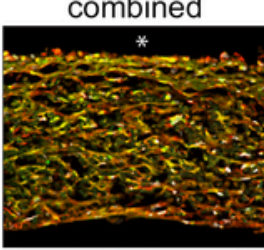

combined

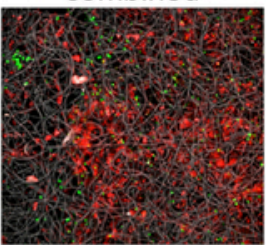

combined

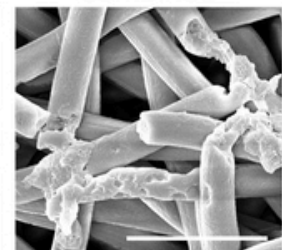

Figure 4: Concept of in situ vascular tissue engineering, cell distribution, tissue production, and scaffold degradation. (A) A schematic illustration depicting the hypothesized phases of scaffold-driven tissue regeneration at the host's functional site. The results shown are derived from experiments that focused on the proliferative phase, in which macrophages and tissue-producing cells have colonized the scaffold material. (B) Myofibroblasts from human saphenous vein (red) and PBMC-derived macrophage (green) distribution at the outer side of the electrospun scaffold (grey) at day 3. Scale bar $200 \mu \mathrm{m}$. (C) Cross-section of the co-culture construct at day 20 stained for collagen type I (green), collagen type III (red), and DAPI (white). Scale bar $100 \mu \mathrm{m}$, *indicates outer side of the construct, corresponding to the flow side. (D) SEM images of decellularized grafts of 8 days macrophage monoculture showing macrophage degradation; scale bar 20 $\mu \mathrm{m}$. Abbreviations: human saphenous venous cells (HVSC); peripheral blood mononuclear cell (PBMC); 4',6-diamidino-2phenylindole (DAPI); scanning electron microscopy (SEM). Panel A was adapted from Wissing and Bonito et al. ${ }^{27}$; panels $B$ and $C$ were adapted from Van Haaften and Wissing et al. ${ }^{44}$; and panel D was adapted from Wissing et al. ${ }^{43}$. Please click here to view a larger version of this figure. 


\section{A.}

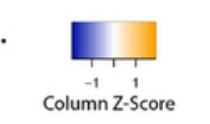

total cytokine secretion $(\mathrm{pg})$

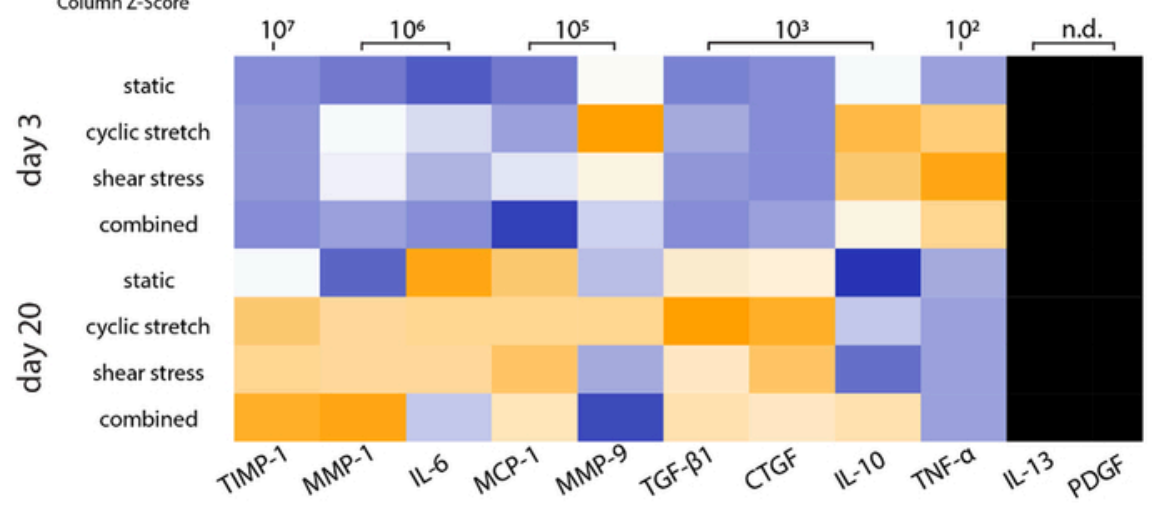

B.
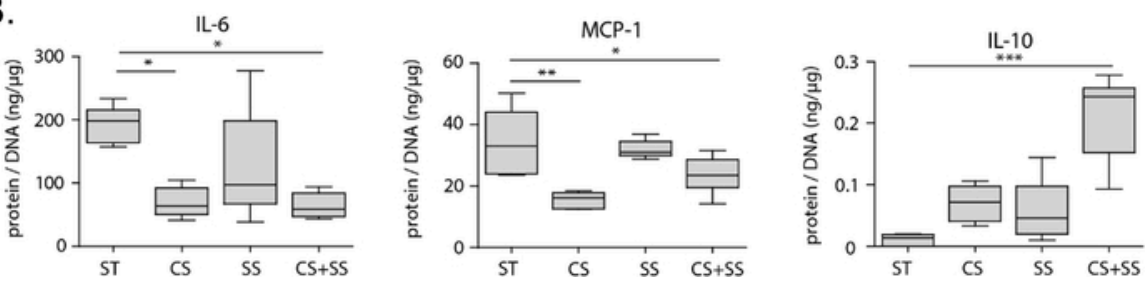

Figure 5: Inflammatory environment in hemodynamically loaded co-culture constructs at 3 days and 20 days. Coculture of human PBMC-derived macrophages and human myofibroblasts from saphenous veins. (A) Heat map of total cytokine secretion measured in supernatant via multiplex ELISA. (B) Boxplots for a selection of cytokines at day 20, normalized to total DNA content. P-values were calculated using Kruskal-Wallis test with a Dunn's multiple comparison test; * $p<0.05,{ }^{* *} p<0.01,{ }^{* *}$ Abbreviations: tissue inhibitor of metalloproteinase (TIMP), matrix metalloproteinase (MMP), interleukin (IL), monocyte chemoattractant protein 1 (MCP-1), transforming growth factor beta 1 (TGF- $\beta 1$ ), connective tissue growth factor (CTGF), tumor necrosis factor alpha (TNF- $\alpha$ ), platelet-derived growth factor (PDGF), static (ST), cyclic stretch (CS), shear stress (SS). Panels A and B were adapted from Van Haaften and Wissing et al. ${ }^{44}$. Please click here to view a larger version of this figure. 
A.
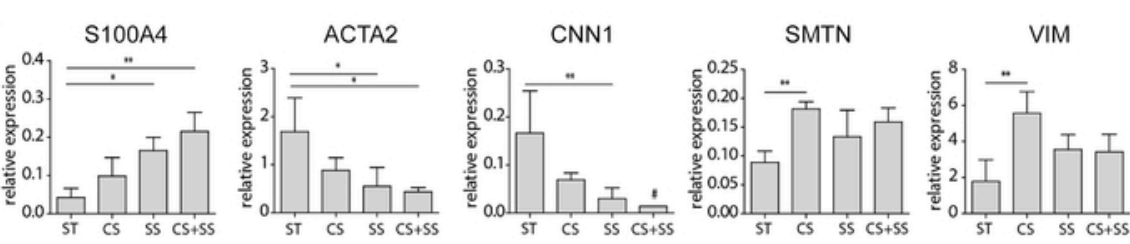

B.

static

cyclic stretch

shear stress

combined
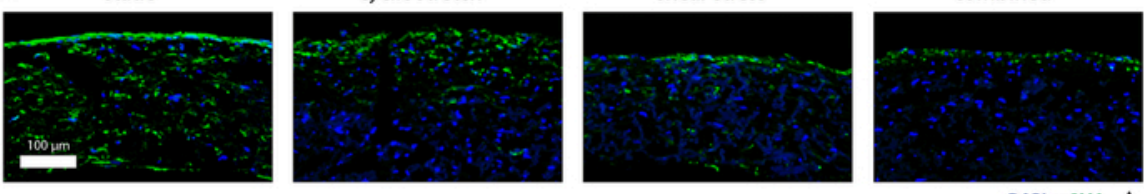

C.
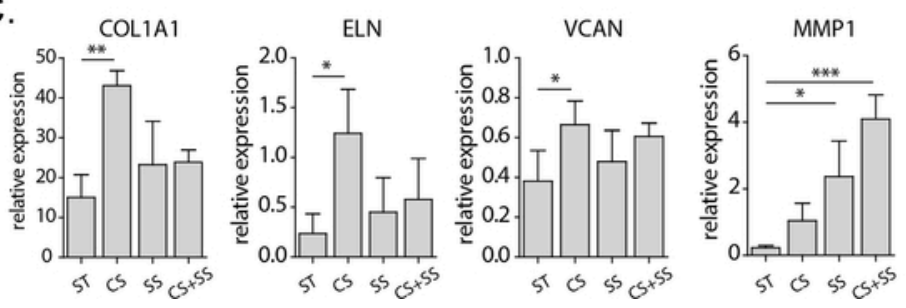

TIMP1

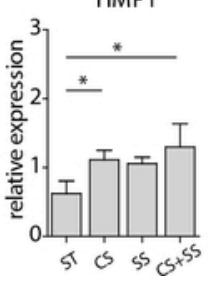

Figure 6: Changes in myofibroblast phenotype and markers of matrix growth and remodeling in response to hemodynamic loading in the vascular constructs at day 20. (A) Relative gene expression of myofibroblast-specific phenotypic markers. (B) Cross-sections stained for aSMA (green) and DAPI (blue), scale bar $100 \mu \mathrm{m}$. (C) Relative expression of genes related to collagenous matrix, elastic matrix, proteoglycans, and remodeling genes. P-values were calculated using Kruskal-Wallis test with a Dunn's multiple comparison test; ${ }^{*} p<0.05,{ }^{* *} p<0.01,{ }^{* * *} p<0.001$. Abbreviations: S100 calcium binding protein A4 (S100A4), alpha-smooth muscle actin (aSMA or ACTA2), calponin 1 (CNN1), smoothelin (SMTN), vimentin (VIM), collagen I (COL1A1), elastin (ELN), versican (VCAN), matrix metalloproteinase (MMP), tissue inhibitor of metalloproteinase (TIMP), static (ST), cyclic stretch (CS), shear stress (SS), 4',6-diamidino-2phenylindole (DAPI). \# measured at or below the detection limit. Panels A, B, and C were adapted from Van Haaften and Wissing et al. ${ }^{44}$. Please click here to view a larger version of this figure.

\section{Supplementary Figure S1: Protein expression myofibroblast- and macrophage-monocultures} subjected to individual and combined hemodynamic loads. (A) Representative confocal images of myofibroblasts, cultured for 10 days with actin fibers (green), nuclei (red), and scaffold (blue), show a clear actin fiber orientation in the loaded samples, when compared to the static samples in which no preferential actin fiber direction can be observed.
Scale bar $50 \mu \mathrm{m}$. (B) Confocal images of same myofibroblast monoculture stained for collagen (green) and nuclei/scaffold (white). Scale bar $50 \mu \mathrm{m}$. (C) Boxplots of protein secretion profiles of statically and dynamically cultured THP1-derived macrophages for 8 days, with calculated M1/M2 ratios based on the cytokine secretion levels of IL-6, TNF- $\alpha$, MCP-1 (proinflammatory) and IL-10, IL-13, MMP-9 (anti-inflammatory). The dot in the MCP-1 graph represents a statistical outlier. (D) 
ELISA data of the relative protein secretion levels of statically and dynamically cultured macrophages at day 8 compared to the average secretion of pro-inflammatory, anti-inflammatory, growth, and remodeling proteins (protein levels were corrected for the average DNA content per group). The dots and shaded areas indicate, respectively, the 50 th and $25^{\text {th }}$ $75^{\text {th }}$ percentiles. Abbreviations: monocyte chemoattractant protein 1 (MCP-1), interleukin (IL), transforming growth factor beta (TGF- $\beta$ ), matrix metalloproteinase (MMP), plateletderived growth factor (PDGF), connective tissue growth factor (CTGF), tumor necrosis factor alpha (TNF- $\alpha$ ), enzyme-linked immunosorbent assay (ELISA). ${ }^{*} p<0.05 ;{ }^{* *} p<0.01$, ${ }^{* * *} p$ $<0.001$. Panels $A$ and $B$ were adapted from Van Haaften et al. ${ }^{19}$. Panels $\mathrm{C}$ and $\mathrm{D}$ were adapted from Wissing et al. ${ }^{43}$. Please click here to download this figure.

\section{Discussion}

The bioreactor described herein allows for the systematic evaluation of the contributions of the individual and combined effects of shear stress and cyclic stretch on inflammation and tissue regeneration in tubular resorbable scaffolds. This approach also enables a large variety of analyses to be performed on vascular constructs, as exemplified in the representative results section. These results show the distinctive impact of the different hemodynamic loading regimes (i.e., different combinations of shear and stretch) on both growth and remodeling of the TEVG construct. These insights, collected via this in vitro platform, aid in the optimization of scaffold design parameters for newly developed in situ TEVGs. To ensure a proper experimental workflow, an understanding of the critical steps and the limitations of this protocol is important.

The most critical steps in the protocol are related to the application of stretch to the samples. For stretch application, it is essential that the setup is leak-free. There are two weak points in the system: the knots mounting the electrospun grafts to the pressure conduits and the connection between the bioreactor base and the flow culture chambers. As described in steps 2.5.2 and 2.5.4, multiple, tight knots have to be positioned exactly at the engraved groove. If the knot is not tight enough or is placed slightly above or below the groove, a slight leakage of hydraulic fluid into the flow culture chamber may occur. This leakage may be detected as a pressure drop in the hydraulic reservoir and a steadily increasing voltage on the strain pump to reach the set pressure value. Besides, this leads to a disturbed flow in the flow culture chamber, an increased risk of contamination of the cell culture, and a dilution of the medium. Once this happens, the flow culture chamber has to be taken out of the experiment, and the screw thread on the hydraulic reservoir should be closed with a white Luer cap. This major measure of taking out a complete sample, which is needed to ensure proper continuation of the experiment for the other seven flow culture chambers, emphasizes the importance of accurately placing tight knots, exactly in the engraved grooves. Only then can proper separation be ensured between the water in the hydraulic reservoir and the medium in the flow culture chambers. To improve robustness of the mounting of the scaffolds, the grooves in the pressure conduits will be made slightly deeper in a revised version of the bioreactor, allowing for easier and better knot placement and thus, secure separation of the hydraulic fluid from the medium.

Another potential source of leakage is between the screw threads of the bioreactor base and the white Luer connectors of the flow culture chamber. Because of possible wear and tear of the Teflon material, an extra silicone O-ring can be added to prevent leakage (step 5.2.3). Moreover, the Teflon bellow of the strain pump should be allowed to 
slightly expand $\mathrm{O} / \mathrm{N}$ one day before the experiment (step 1.4). If leakage occurs, the lost hydraulic fluid should be compensated by adding a small amount of ultrapure water via one of the eight screw threads (use a syringe with needle and flexible wire). Place the flow culture chamber back with a small piece of parafilm between the screw thread and the white Luer connector on the bottom compartment of the flow culture chamber. To overcome this leakage issue in future experiments, the current Teflon screw threads on the bioreactor base will be replaced by stainless steel threads in the next-generation version of the bioreactor to prevent wearout of the system.

The variation in stretch (Figure 3C) is larger than the variation in WSS (Figure 3D), as stretch is more difficult to control. Nevertheless, besides the measures to prevent leakage, there are other measures that limit the variation in stretch: (i) avoiding air bubbles in the flute (step 1.4 and 5.2.1), (ii) ensuring consistent pre-stretch among the different samples (step 2.5.3), and (iii) ensuring consistent scaffold properties among different samples (step 1.3).

Finally, extra caution is needed when mounting the electrospun scaffold onto the silicone tubing (step 2.3). Specifically, when the fragile electrospun scaffold needs to be pulled over the stretched silicone tubing, it is important not to apply too much force to prevent the electrospun graft from being damaged, especially on the inside of the electrospun graft. If damage occurs on the inside of the electrospun graft, either from forceful pulling or from too weak stretching of the silicone tube, the extent of the damage to the electrospun fibers only becomes visible after the construct is harvested and analyzed. In the current setup, the success of the seeding can only be confirmed upon immunofluorescence or immunohistochemical analysis, after sacrificing the sample. However, the seeding procedure with fibrin as the cell carrier is a well-established method ${ }^{67}$ that typically leads to a homogenous cell distribution for scaffolds with a sufficiently large pore size. One of the most important aspects with respect to cell seeding is to ensure that the scaffold is as dry as possible prior to seeding (step 4.4), to prevent the fibrin gel with the cells from passing through the wet scaffold, resulting in inhomogeneous seeding. Finally, although the decontamination method of the electrospun graft by UV radiation and dipping in $30 \%$ ethanol is not as stringent as the sterilization of electrospun grafts prepared for in vivo studies, which are often sterilized by ethylene oxide or gamma irradiation, it is sufficient for in vitro culture experiments that can last up to 20 days without any signs of contamination (for examples, see Figure 3, Figure 4, Figure 5, Figure 6, and Van Haaften and Wissing $(2020)^{44}$ ). Moreover, the PCL-BU material that is used here, does not allow for long exposure to high ethanol concentrations. The most suitable sterilization method can be chosen depending on the material used.

In addition to the results of previously performed coculture studies, a broader variety of studies can be performed with the same system. The system was previously employed to perform dynamic monocultures of myofibroblasts (Supplementary Figure 1A-B) and macrophages (Supplementary Figure 1C-D) to investigate the effects of hemodynamic loading on individual cell types and their paracrine signaling ${ }^{19,43}$. Different hemodynamic loading regimes resulted in clear actin fiber orientation in myofibroblasts (Supplementary Figure 1A) and distinctly different collagen deposition (Supplementary Figure 1B) after 10 days. The cytokine production by THP1-derived macrophages was drastically different between the different hemodynamic loads (Supplementary Figure 1C) and showed a more pro-inflammatory profile when loaded 
(Supplementary Figure 1D). Other validated possibilities include the application of oscillatory flow, by using an extra pump and fluidic unit. The viscosity of the medium can be increased towards the range of blood viscosity (e.g., by adding xanthan gum $)^{69}$. Modulating the medium viscosity represents an additional variable to broaden the range of applicable shear stresses. Lastly, although the described protocol employs the 'Ibidi' flow-conditioning setup, setups from other manufacturers can be used as well, as long as similar flow regimes can be applied.

One of the major advantages of using this bioreactor system is the relatively large construct (approximately $15 \mathrm{~mm} \times 10.5$ $\mathrm{mm}$ ) that can be hemodynamically loaded, allowing for a wide variety of possible readout parameters to be extracted from a single sample. At the same time, the construct size may be viewed as a limitation as well, as this setup requires a relatively large amount of (sometimes costly) material, especially if primary cells are used, or if the culture medium requires costly additives. Moreover, the throughput of the setup is relatively low. Consequently, the current setup is particularly suited for hypothesis-driven research in which a limited number of variables is comprehensively tested, rather than the screening of a large number of variables with limited readout. For future experiments, small improvements on the current setup are being made to enable the option for mounting smaller scaffolds and downscaling the size of the medium reservoirs. With respect to the latter, the current volume of the medium reservoirs is required to enable sufficient volumetric flow rates to achieve the desired shear stresses. The required flow rates-and with that, the volume of the medium reservoirs-can be reduced by increasing the viscosity of the medium (e.g., by adding xanthan gum, as previously established ${ }^{69}$ ).
To conclude, this bioreactor allows for the quantification of the individual and combined effects of shear stress and cyclic stretch on tissue growth and remodeling on a wide variety of elastomeric 3D biomaterial scaffolds. The bioreactor can culture up to eight vascular constructs under various loading conditions. Owing to its design, the bioreactor is especially suited to study the interplay between hemodynamics and in situ vascular TE processes.

\section{Disclosures}

The authors have nothing to disclose.

\section{Acknowledgments}

This study is financially supported by ZonMw as part of the LSH 2Treat program (436001003) and the Dutch Kidney Foundation (14a2d507). N.A.K. acknowledges support from the European Research Council (851960). We gratefully acknowledge the Gravitation Program "Materials Driven Regeneration", funded by the Netherlands Organization for Scientific Research (024.003.013).

\section{References}

1. Chlupác, J., Filová, E., Bacáková, L. Blood vessel replacement: 50 years of development and tissue engineering paradigms in vascular surgery. Physiological Research. 58 (Suppl 2), S119-39 (2009).

2. Huygens, S. A. et al. Bioprosthetic aortic valve replacement in elderly patients: Meta-analysis and microsimulation. The Journal of Thoracic and Cardiovascular Surgery. 157 (6), 2189-2197.e14 (2019).

3. Huygens, S. A. et al. Contemporary outcomes after surgical aortic valve replacement with bioprostheses and allografts: a systematic review and meta-analysis. 
European Journal of Cardio-Thoracic Surgery. 50 (4), 605-616 (2016).

4. Loh, S. A. et al. Mid- and long-term results of the treatment of infrainguinal arterial occlusive disease with precuffed expanded polytetrafluoroethylene grafts compared with vein grafts. Annals of Vascular Surgery. 27 (2), 208-217 (2013).

5. Tara, S. et al. Vessel bioengineering. Circulation Journal. 78 (1), 12-19 (2014).

6. Huang, A. H., Niklason, L. E. Engineering of arteries in vitro. Cellular and Molecular Life Sciences. 71 (11), 2103-2118 (2014).

7. Bouten, C. V. C., Smits, A. I. P. M., Baaijens, F. P. T. Can we grow valves inside the heart? Perspective on material-based in situ heart valve tissue engineering. Frontiers in Cardiovascular Medicine. 5, 54 (2018).

8. Fioretta, E. S. et al. Next-generation tissue-engineered heart valves with repair, remodelling and regeneration capacity. Nature Reviews Cardiology. (2020).

9. Kirkton, R. D. et al. Bioengineered human acellular vessels recellularize and evolve into living blood vessels after human implantation. Science Translational Medicine. 11 (485), eaau6934 (2019).

10. Gutowski, P. et al. Arterial reconstruction with human bioengineered acellular blood vessels in patients with peripheral arterial disease. Journal of Vascular Surgery. (2020).

11. Syedain, Z. et al. Tissue engineering of acellular vascular grafts capable of somatic growth in young lambs. Nature Communications. 7 (12951), 12951 (2016).

12. Sugiura, T. et al. Tissue-engineered vascular grafts in children with congenital heart disease: intermediate term follow-up. Seminars in Thoracic and Cardiovascular Surgery. 30 (2), 175-179 (2018).

13. Kluin, J. et al. In situ heart valve tissue engineering using a bioresorbable elastomeric implant - From material design to 12 months follow-up in sheep. Biomaterials. 125, 101-117 (2017).

14. Fioretta, E. S. et al. Differential leaflet remodeling of bone marrow cell pre-seeded versus nonseeded bioresorbable transcatheter pulmonary valve replacements. JACC. Basic to Translational Science. $\mathbf{5}$ (1), 15-31 (2020).

15. Van Haaften, E. E., Bouten, C. V. C., Kurniawan, N. A. Vascular mechanobiology: towards control of in situ regeneration. Cells. 1-24 (2017).

16. De Jonge, N. et al. Matrix production and organization by endothelial colony forming cells in mechanically strained engineered tissue constructs. PLOS ONE. 8 (9), e73161 (2013).

17. Schmidt, J. B., Chen, K., Tranquillo, R. T. Effects of intermittent and incremental cyclic stretch on ERK signaling and collagen production in engineered tissue. Cellular and Molecular Bioengineering. 9 (1), 55-64 (2016).

18. Luo, J. et al. Tissue-engineered vascular grafts with advanced mechanical strength from human iPSCs. Cell Stem Cell. 26 (2), 251-261.e8 (2020).

19. Van Haaften, E. E. et al. Decoupling the effect of shear stress and stretch on tissue growth and remodeling in a vascular graft. Tissue Engineering Part C: Methods. 24 (7), 418-429 (2018).

20. Gupta, V., Tseng, H., Lawrence, B. D., Jane GrandeAllen, K. Effect of cyclic mechanical strain on 
glycosaminoglycan and proteoglycan synthesis by heart valve cells. Acta Biomaterialia. 5 (2), 531-540 (2009).

21. Lin, S., Mequanint, K. Bioreactor-induced mesenchymal progenitor cell differentiation and elastic fiber assembly in engineered vascular tissues. Acta Biomaterialia. 59, 200-209 (2017).

22. Venkataraman, L., Bashur, C. A., Ramamurthi, A. Impact of cyclic stretch on induced elastogenesis within collagenous conduits. Tissue Engineering. Part A. 20 (9-10), 1403-15 (2014).

23. Huang, A. H. et al. Biaxial stretch improves elastic fiber maturation, collagen arrangement, and mechanical properties in engineered arteries. Tissue Engineering Part C Methods. 22 (6), 524-533 (2016).

24. Hinderer, S. et al. In vitro elastogenesis: instructing human vascular smooth muscle cells to generate an elastic fiber-containing extracellular matrix scaffold. Biomedical Materials. 10 (3), 034102 (2015).

25. Eoh, J. H. et al. Enhanced elastin synthesis and maturation in human vascular smooth muscle tissue derived from induced-pluripotent stem cells. Acta Biomaterialia. 52, 49-59 (2017).

26. Smits, A. I. P. M., Bouten, C. V. C. Tissue engineering meets immunoengineering: Prospective on personalized in situ tissue engineering strategies. Current Opinion in Biomedical Engineering. 6, 17-26 (2018).

27. Wissing, T. B., Bonito, V., Bouten, C. V. C., Smits, A. I. P. M. Biomaterial-driven in situ cardiovascular tissue engineering-a multi-disciplinary perspective. npj Regenerative Medicine. 2 (1), 18 (2017).

28. Hibino, N. et al. A critical role for macrophages in neovessel formation and the development of stenosis in tissue-engineered vascular grafts. The FASEB Journal. 25 (12), 4253-63 (2011).

29. Godwin, J. W., Pinto, A. R., Rosenthal, N. A. Macrophages are required for adult salamander limb regeneration. Proceedings of the National Academy of Sciences of the United States of America. 110 (23), 9415-20 (2013).

30. Godwin, J. W., Debuque, R., Salimova, E., Rosenthal, N. A. Heart regeneration in the salamander relies on macrophage-mediated control of fibroblast activation and the extracellular landscape. npj Regenerative Medicine. 2 (1), 22 (2017).

31. McBane, J. E., Cai, K., Labow, R. S., Santerre, J. P. Coculturing monocytes with smooth muscle cells improves cell distribution within a degradable polyurethane scaffold and reduces inflammatory cytokines. Acta Biomaterialia. 8 (2), 488-501 (2012).

32. Battiston, K. G., Ouyang, B., Labow, R. S., Simmons, C. A., Santerre, J. P. Monocyte/macrophage cytokine activity regulates vascular smooth muscle cell function within a degradable polyurethane scaffold. Acta Biomaterialia. 10 (3), 1146-1155 (2014).

33. Ploeger, D. T. et al. Cell plasticity in wound healing: paracrine factors of $\mathrm{M} 1 / \mathrm{M} 2$ polarized macrophages influence the phenotypical state of dermal fibroblasts. Cell Communication and Signaling. 11 (1), 29 (2013).

34. McBane, J. E., Santerre, J. P., Labow, R. S. The interaction between hydrolytic and oxidative pathways in macrophage-mediated polyurethane degradation. Journal of Biomedical Materials Research. Part A. 82 (4), 984-94 (2007).

35. Wissing, T. B. et al. Macrophage-driven biomaterial degradation depends on scaffold microarchitecture. 
Frontiers in Bioengineering and Biotechnology. 7, 87 (2019).

36. Wolf, M. T., Vodovotz, Y., Tottey, S., Brown, B. N., Badylak, S. F. Predicting in vivo responses to biomaterials via combined in vitro and in silico analysis. Tissue Engineering Part C: Methods. 21 (2), 148-159 (2015).

37. Grotenhuis, N., Bayon, Y., Lange, J. F., Van Osch, G. J. V. M., Bastiaansen-Jenniskens, Y. M. A culture model to analyze the acute biomaterial-dependent reaction of human primary macrophages. Biochemical and Biophysical Research Communications. 433 (1), 115-120 (2013).

38. Jannasch, M. et al. A comparative multi-parametric in vitro model identifies the power of test conditions to predict the fibrotic tendency of a biomaterial. Scientific Reports. 7 (1), 1689 (2017).

39. Wang, Z. et al. The effect of thick fibers and large pores of electrospun poly( $\varepsilon$-caprolactone) vascular grafts on macrophage polarization and arterial regeneration. Biomaterials. 35 (22), 5700-5710 (2014).

40. McWhorter, F. Y., Davis, C. T., Liu, W. F. Physical and mechanical regulation of macrophage phenotype and function. Cellular and Molecular Life Sciences. 72 (7), 1303-1316 (2014).

41. Ballotta, V., Driessen-Mol, A., Bouten, C. V. C., Baaijens, F. P. T. Strain-dependent modulation of macrophage polarization within scaffolds. Biomaterials. 35 (18), 4919-28 (2014).

42. Dziki, J. L. et al. The effect of mechanical loading upon extracellular matrix bioscaffold-mediated skeletal muscle remodeling. Tissue Engineering. Part A. 24 (1-2), 34-46 (2018).
43. Wissing, T. B. et al. Hemodynamic loads distinctively impact the secretory profile of biomaterial-activated macrophages - implications for in situ vascular tissue engineering. Biomaterials Science. 8 (1), 132-147 (2020).

44. Van Haaften, E. E., Wissing, T. B., Kurniawan, N. A., Smits, A. I. P. M., Bouten, C. V. C. Human in vitro model mimicking material-driven vascular regeneration reveals how cyclic stretch and shear stress differentially modulate inflammation and matrix deposition. Advanced Biosystems. 4 (6), 1900249 (2020).

45. Ballotta, V., Smits, A. I. P. M., Driessen-Mol, A., Bouten, C. V. C., Baaijens, F. P. T. Synergistic protein secretion by mesenchymal stromal cells seeded in $3 D$ scaffolds and circulating leukocytes in physiological flow. Biomaterials. 35 (33), 9100-13 (2014).

46. Bonito, V., de Kort, B. J., Bouten, C. V. C., Smits, A. I. P. M. Cyclic strain affects macrophage cytokine secretion and extracellular matrix turnover in electrospun scaffolds. Tissue Engineering Part A. 25 (17-18), 1310-1325 (2019).

47. Battiston, K. G., Labow, R. S., Simmons, C. A., Santerre, J. P. Immunomodulatory polymeric scaffold enhances extracellular matrix production in cell co-cultures under dynamic mechanical stimulation. Acta Biomaterialia. 24 74-86 (2015).

48. Smits, A. I. P. M., Driessen-Mol, A., Bouten, C. V. C., Baaijens, F. P. T. A mesofluidics-based test platform for systematic development of scaffolds for in situ cardiovascular tissue engineering. Tissue Engineering Part C: Methods. 18 (6), 475-485 (2012).

49. Smits, A. I. P. M., Ballotta, V., Driessen-Mol, A., Bouten, C. V. C., Baaijens, F. P. T. T. Shear flow affects selective 
monocyte recruitment into MCP-1-loaded scaffolds. Journal of Cellular and Molecular Medicine. 18 (11), 2176-88 (2014).

50. Ballotta, V., Smits, A. I. P. M., Driessen-Mol, A., Bouten, C. V. C., Baaijens, F. P. T. Synergistic protein secretion by mesenchymal stromal cells seeded in 3D scaffolds and circulating leukocytes in physiological flow. Biomaterials. 35 (33), 9100-9113, (2014).

51. Fahy, N., Menzel, U., Alini, M., Stoddart, M. J. Shear and dynamic compression modulates the inflammatory phenotype of human monocytes in vitro. Frontiers in Immunology. 10, 383 (2019).

52. Pennings, I. et al. Layer-specific cell differentiation in bi-layered vascular grafts under flow perfusion. Biofabrication. 12 (1), 015009 (2019).

53. Wang, J. et al. Ex vivo blood vessel bioreactor for analysis of the biodegradation of magnesium stent models with and without vessel wall integration. Acta Biomater. 50, 546-555 (2017).

54. Huang, A. H. et al. Design and use of a novel bioreactor for regeneration of biaxially stretched tissue-engineered vessels. Tissue Engineering. Part C, Methods. 21 (8), 841-51 (2015).

55. Huang, A. H., Niklason, L. E. Engineering biologicalbased vascular grafts using a pulsatile bioreactor. Journal of Visualized Experiments. (52) (2011).

56. Bono, N. et al. A Dual-mode bioreactor system for tissue engineered vascular models. Annals of Biomedical Engineering. 45 (6), 1496-1510 (2017).

57. Wolf, F. et al. VascuTrainer: a mobile and disposable bioreactor system for the conditioning of tissue- engineered vascular grafts. Annals of Biomedical Engineering. 46 (4), 616-626 (2018).

58. Ramaswamy, S. et al. A novel bioreactor for mechanobiological studies of engineered heart valve tissue formation under pulmonary arterial physiological flow conditions. Journal of Biomechanical Engineering. 136 (12), 121009 (2014).

59. Piola, M. et al. A compact and automated ex vivo vessel culture system for the pulsatile pressure conditioning of human saphenous veins. Journal of Tissue Engineering and Regenerative Medicine. 10 (3), E204-E215 (2016).

60. Vanerio, N., Stijnen, M., de Mol, B. A. J. M., Kock, L. M. An innovative ex vivo vascular bioreactor as comprehensive tool to study the behavior of native blood vessels under physiologically relevant conditions. Journal of Engineering and Science in Medical Diagnostics and Therapy. 2 (4) (2019).

61. Kural, M. H., Dai, G., Niklason, L. E., Gui, L. An ex vivo vessel injury model to study remodeling. Cell Transplantation. 27 (9), 1375-1389 (2018).

62. Sinha, R. et al. A medium throughput device to study the effects of combinations of surface strains and fluid-flow shear stresses on cells. Lab on a Chip. 15 (2), 429-439 (2015).

63. Beca, B. M., Sun, Y., Wong, E., Moraes, C., Simmons, C. A. Dynamic bioreactors with integrated microfabricated devices for mechanobiological screening. Tissue Engineering Part C: Methods. 25 (10), 581-592 (2019).

64. Liu, H., Usprech, J., Sun, Y., Simmons, C. A. A microfabricated platform with hydrogel arrays for 3D mechanical stimulation of cells. Acta Biomaterialia. 34, 113-124 (2016). 
65. Szafron, J. M., Ramachandra, A. B., Breuer, C. K., Marsden, A. L., Humphrey, J. D. Optimization of tissueengineered vascular graft design using computational modeling. Tissue Engineering Part C: Methods. 25 (10), 561-570 (2019).

66. Emmert, M. Y. et al. Computational modeling guides tissue-engineered heart valve design for long-term in vivo performance in a translational sheep model. Science Translational Medicine. 10 (440) (2018).

67. Mol, A. et al. Fibrin as a cell carrier in cardiovascular tissue engineering applications. Biomaterials. 26 (16), 3113-21 (2005).

68. van Kelle, M. A. J. et al. A Bioreactor to identify the driving mechanical stimuli of tissue growth and remodeling. Tissue Engineering Part C: Methods. 23 (6), 377-387 (2017).

69. van den Broek, C. N. et al. Medium with blood-analog mechanical properties for cardiovascular tissue culturing. Biorheology. 45 (6), 651-61 (2008). 